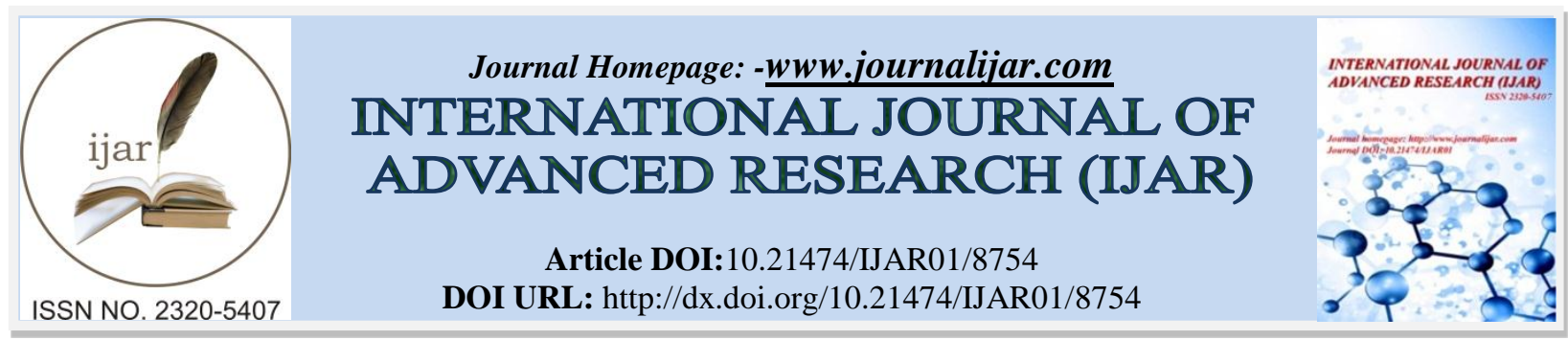

RESEARCH ARTICLE

\title{
EVALUATION AND COMPARISON OF THE TENSILE BOND STRENGTH OF TWO DIFFERENT LUTING AGENTS ON AN ENDODONTIC FIBER POST AFTER TWO DIFFERENT SURFACE TREATMENTS - AN IN VITRO STUDY.
}

\author{
Nila S Das ${ }^{1}$, Rupesh P L ${ }^{2}$,Bharat Kataraki ${ }^{3}$, Basavaraj S Salagundi ${ }^{4}$, Unni Pympallil ${ }^{3}$, Arundati N. Raj ${ }^{5}$ and \\ Vaishnavi Gowda ${ }^{1}$. \\ 1. BDS, MDS, Post Graduate, Department of Prosthodontics,Coorg Institute of Dental Sciences, Virajpet. \\ 2. MDS, Proffesor and Chair, Department of Prosthodontics, Coorg Institute of Dental Sciences, Virajpet. \\ 3. MDS, Senior Lecturer, Department of Prosthodontics, Coorg Institute of Dental Sciences, Virajpet. \\ 4. MDS, Proffesor and Head, Department of Prosthodontics, Coorg Institute of Dental Sciences, Virajpet. \\ 5. MDS, Professor, Department of Prosthodontics, Coorg Institute of Dental Sciences, Virajpet.
}

\section{Manuscript Info}

\section{Manuscript History}

Received: 20 January 2019

Final Accepted: 22 February 2019

Published: March 2019

Key words:-

Tensile bond strength; Glass fiber post; resin cement.

\begin{abstract}
Purpose:To evaluate and compare tensile bond strength of two different luting agents on an endodontic fiber post after two different surface treatments.

Methodology: 60 mandibular first premolar freshly extracted teeth were used. The root canals were prepared using a step-back technique and obturated. The roots were divided into 2 experimental groups based on the luting cements (Rely X U200 \& Calibra) and further divided into 3 subgroups according to surface treatments used $(n=10$, subgroups are A- control, B- Laser, C- Sandblasting. After post cementation the specimens were cut in to three 2-mm-thick segments (1 apical, 1 middle, and 1 cervical) were prepared. The tensile bond strength was measured using a universal testing machine. The data was analysed using the independent $t$ test and one way ANOVA test followed by Post Hoc Tukey's test.

Conclusion: Sandblasting of glass fiber post provided higher tensile bond strength values for Calibra cement hence it can be recommended as a surface treatment method suitable for glass fiber post and Calibra is the cement of choice.
\end{abstract}

Copy Right, IJAR, 2019,. All rights reserved.

\section{Introduction:-}

Endodontically treated teeth may require extensive coronal reconstruction and, depending on the severity of the coronal tissue loss, intracanal posts are widely used for restoring endodontically treated teeth with insufficient coronal tooth structure to achieve retention to the core and for the definitive restoration. ${ }^{1,2}$ Posts made of metal alloys were reported to have less retention, causes serious types of root fractures, that is the rigid metal posts can defy to the lateral forces without deformation because of the homogeneous structure. However, this rigidity causes root fractures according to the stress distribution and they compromise esthetics and are also susceptible to corrosion. The choice of materials used in these cases has changed from rigid materials, such as gold and zircon dioxide, to materials that have mechanical characteristics that more closely resemble dentin, such as fiber posts and

Corresponding Author:-Nila S Das.

Address:-BDS, MDS, Post Graduate, Department of Prosthodontics,Coorg Institute of Dental Sciences, Virajpet. 
composite resins. Prefabricated post systems have recently become more popular because they can provide satisfactory results, while saving time and reducing costs. However, a prefabricated post should adequately adapt to the prepared root canal; otherwise, a cast post and core should be used. Passive, tapered posts have a shape that is congruent with the root canal configuration and allow for optimal preservation of tooth structure, especially in the apical region. Use of these materials diminishes the probability of root fracture, because these failures occur particularly in the post, allowing for tooth recovery. Many invitro studies have investigated factors that may affect the retention of a post, which may include design, length, diameter and surface treatments of the posts. ${ }^{1,2,3,4}$ To improve the fracture resistance of endodontically treated teeth and to obtain esthetic restorations, fiber-reinforced composite (FRC) posts were chosen. FRC posts are composed of carbon, quartz, zirconia, glass, or silica fibers that are surrounded by polymer resin matrix. FRC post systems approximately have a ratio of fiber at 35 and $65 \%$. High ratio of fiber content is typically more rigid and durable. Due to FRC posts elastic modulus, which is similar to that of dentin, they can produce a favorable stress distribution and high success rates without the occurrence of root fractures. Posts made of tooth-colored material, such as glass fibers or zirconia ceramics, have become popular because they increase the transmission of light within the root and overlying gingival tissues. The low modulus of elasticity of fiber-reinforced epoxy resin posts has been purported to reduce the risk of root fracture. In addition, the restoration of endodontically treated teeth with metal-free materials eliminates the potential hazards of corrosion and allergic hypersensitivity. Glass-fiber posts are composed of glass fibers, inorganic filler, and a resin matrix. They are usually luted with a resin cement to increase their retention and improve the mechanical performance of the restored teeth. Fiber-reinforced posts also have the advantage of easy removal if endodontic retreatment is required. Use of these materials diminishes the probability of root fracture, because these failures occur particularly in the post, allowing for tooth recovery. ${ }^{2,4,5}$ Several factors affect the retention of FRC post within root canals, such as time of post space preparation and cementation, type of post and its adaptation to the post space, type of endodontic cement, adhesive and cementation system, and operative procedures. ${ }^{1}$ In many studies, different surface treatments were tested to investigate the bond strength between a composite resin matrix and resin cement. ${ }^{2}$ Surface treatment has become popular, which improves the adhesion properties of a material by facilitating their chemical and micromechanical bonding between different units. It has been shown that prior to the cementation process, the preparations on the post surface affect the bond strength of post and cement interface. The polymer matrix in the structure of FRC post is unable to react with the monomers of resin cements. On the basis of this fact, it has been suggested to apply surface preparation processes that selectively react with the epoxy resin matrix of the FRC post in order to roughen the post surface and expose the glass fibers. These procedures allow the micro-mechanical interlocking of the adhesive/cement with the post. Chemical interaction that forms the bond between fiber post and core is not sufficient enough to withstand the occlusal stresses, surface pre-treatment of fiber post is the common method employed to improve the adhesion property of material. Chemical interaction between the fiber post surface and the composite can only ensure the bond at the post-core interfacial level. Several surface treatments have been suggested in order to increase the present bonding with fiber post. These procedures fall into three categories: 1) Coating with priming solutions i.e. Treatments that result in chemical bonding between a composite and post; 2) Sand blasting and Etching i.e. Treatments that intend to roughen the post surface or 3) Combination of micromechanical and chemical components either by using the above mentioned two methods or a unique system \{such as Co-Jet\}. Sandblasting of the dental restorations is often used to clean the materials, to increase the surface area, and to ensure the micro-retentive areas. In this sense, air abrasion is used for FRC posts to remove the superficial layer of resinous matrix and to create micro-retentive areas on the post surfaces in order to improve the bond strength between FRC posts and resin cements. Recently, lasers become popular in dentistry. Laser technology is being developed very quickly and is beginning to be used in the different fields of dentistry. A new type of laser called Er:YAG emitted in the wavelength of 2,940 $\mathrm{nm}$ matches exactly the maximal absorption in water. Also, well absorbed in hydroxyapatite, this laser seems to have been made for the effective removal of dentin and enamel with only minor side-effects such as thermal damage. The potential of Er:YAG lasers for the ablation of hard tissue in dentistry was demonstrated already in 1989. Many of the technological advances have been directed at the use of lasers in clinical settings as an alternative to the acid etching of dental materials or teeth for improving bond strength. ${ }^{3,4}$ The bonding performance of resin cements is dependent on the quality of the hybrid layer. Some factors such as dentin morphology, bonding system, and luting cement and its cure may interfere with hybrid layer formation along the root canal walls, affecting post retention. This hybridization is critical in the apical third of the post space because of difficulty in establishing adhesion in this area. ${ }^{5}$ In addition, roughening the prepared dentinal surface increased the retention of posts or artificial crowns by increasing the surface area and enhancing the mechanical interlocking between the dentin surface and the cement. The effect of the cement type on retention of the post and on fracture resistance of endodontically treated teeth has been investigated extensively. The use of resin cement has been found to significantly increase retention of posts and fracture resistance of the tooth compared with 
other cements. ${ }^{2}$ The cement has a lower modulus of elasticity then two materials it joins and thus a zone of highly concentrated load and stresses is created as we previously described in a study these considerations suggest that composite resin which has mechanical characteristics similar to dentin would be a better material to use in the critical interface between post and dentin especially when cement thickness exceeds $500 \mu \mathrm{m}^{7}$ These materials can be light polymerized or dual cured. Recently, a self-adhesive resin cement that requires no dentin pretreatment and has a dual-cure mechanism was introduced on the dental market. ${ }^{1}$ Hence the present study was aimed to evaluate and compare the tensile bond strength of 2 different luting agents on an endodontic fiber post after two different surface treatments.

\section{Methodology:-}

Sixty mandibular first premolar freshly extracted in connection with orthodontic treatments, with straight root canals, anatomically similar root segments, and fully developed apices, were selected (figure 3). All external debris were removed with an ultrasonic scaler, and the teeth were stored in saline solution when not under testing. The specimens are then cut perpendicular to the long axis after marking $14 \mathrm{~mm}$ from the apex of the tooth towards the occlusal surface (figure 4,5), to standardize the length, using a slow-speed diamond disc. The pulp tissue was removed with a barbed broach. Canal patency was determined by passing a file (size $10 \mathrm{~K}$-file) after the pulp is removed. Canal working lengths were established $1.0 \mathrm{~mm}$ short of the apical foramina (i.e. $13 \mathrm{~mm}$ ). A step-back technique was used for canal instrumentation (figure 6). The same operator was instrumented all root canals to the same size (size 55 file). During instrumentation, canals were irrigated with $1 \mathrm{ml}$ of $5.25 \% \mathrm{NaOCl}$. Upon completion of the instrumentation, the specimens were divided into 6 groups of 10 each. Before obturation, the root canals were dried with paper points and obturated with lateral condensation of gutta-percha with a sealer (figure 9). A sealer was introduced into root canals using a spreader instrument. Gutta-percha points will then be coated with the sealer and placed in the root canals up to the working length and the gutta-percha filled root canals were stored in deionized water for $24 \mathrm{hrs}$ at $37^{\circ} \mathrm{C}$ (figure 11). Endo fiber posts of $1.5 \mathrm{~mm}$ diameter was selected and each post was marked at $10 \mathrm{~mm}$ from its apical end. A line was drawn around the post at this point and all posts were cut at that point using a water cooled diamond rotary cutting instrument. Gutta-percha was removed to create post space from the root canal with Peeso Reamers after marking $10 \mathrm{~mm}$ on the reamer using a stopper. The manufacturer's recommended instruments were used to clear the post space (figure 9).

The specimens were categorized into groups I and group II according to the cements used for luting, and further sub grouped into sub group A, B, C according to the surface treatment protocol.

1. Group I: Fiber posts luted with Rely-X cement (Self adhesive dual cure resin cement)

2. Group IA: Fiber post without surface treatment luted with Rely-X cement

3. Group IB: Fiber post with Er: YAG laser treatment $(150 \mathrm{~mJ}$ at $10 \mathrm{~Hz}$ for $20 \mathrm{~s}$ at $100 \mu \mathrm{s}$ pulse duration) luted with Rely-X cement (figure21)

4. Group IC: Fiber post with sand blasting $(150 \mu \mathrm{m}$ aluminium oxide was blasted for $20 \mathrm{~s}$ at an operating distance of $10 \mathrm{~mm}$ from the post) luted with Rely-X cement (figure 16, 17, 18)

5. Group II: Fiber post luted with Calibra cement (Esthetic dual cure resin cement)

6. Group IIA: Fiber post without surface treatment luted with Calibra cement

7. Group IIB: Fiber post with Er:YAG laser treatment $(150 \mathrm{~mJ}$ at $10 \mathrm{~Hz}$ for $20 \mathrm{~s}$ at $100 \mu \mathrm{s}$ pulse duration) luted with Calibra cement

8. Group II C: Fiber post with sand blasting $(150 \mu \mathrm{m}$ aluminium oxide was blasted for $20 \mathrm{~s}$ at an operating distance of $10 \mathrm{~mm}$ from the post) luted with Calibra cement

The posts were then cemented using the cements Rely X-U200 and Calibra (figure 14, 15) mix according to the manufacturer's instruction. The mixed cement was applied on to the post spaces using a spreader instrument. The posts will be then coated with the cement and slowly seated by using finger pressure into the post space. Excess cement was removed with an explorer. Once the cement was set the specimens were stored in saline for $24 \mathrm{hrs}$ at $37^{\circ} \mathrm{C}$. Each root $(\mathrm{n}=10)$ was sectioned horizontally with a slow-speed diamond disc to produce three $2 \mathrm{~mm}$ thick segments (1 apical, 1 middle, and 1 cervical) (Figure 21). All specimens were subjected to testing for the push out bond strength using the universal testing machine. The post was loaded with a $1 \mathrm{~mm}$ diameter cylindrical plunger. The plunger tip was sized and positioned to touch only the post, without stressing the surrounding post space walls. The load was applied on the apical aspect of the root slice and in an apical-coronal direction, so as to push the post toward the larger part of the root slice, thus avoiding any limitation to the post movement owing to the post space taper (Fig.2). Loading was performed on a universal testing machine at a cross-head speed of $1.0 \mathrm{~mm} / \mathrm{min}$ until 
bond failure occurred (figure $-22,23,24)$. The force $(\mathrm{N})$ required to debond the post from the dentin disc was recorded for all posts.

Fig 1:-Schematic view of preparation of specimen preparation for push-out test

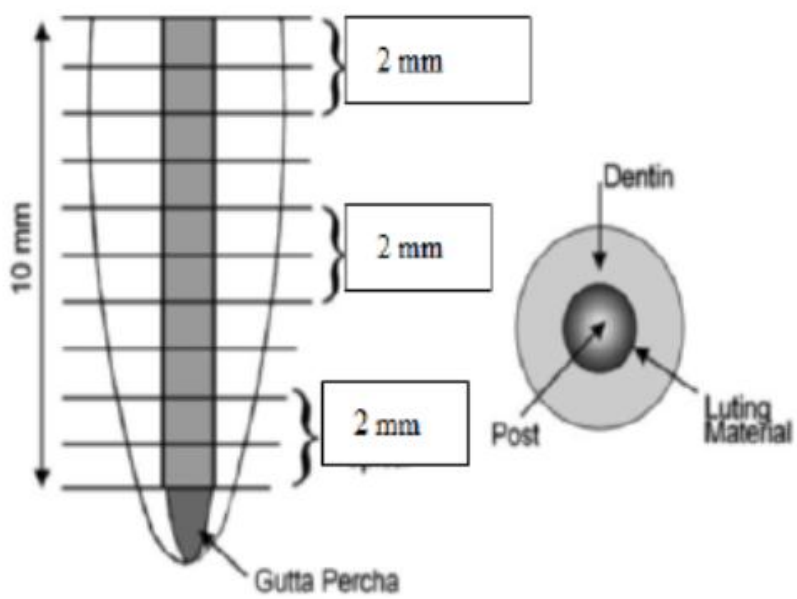

Fig 2:-Push-out test device.

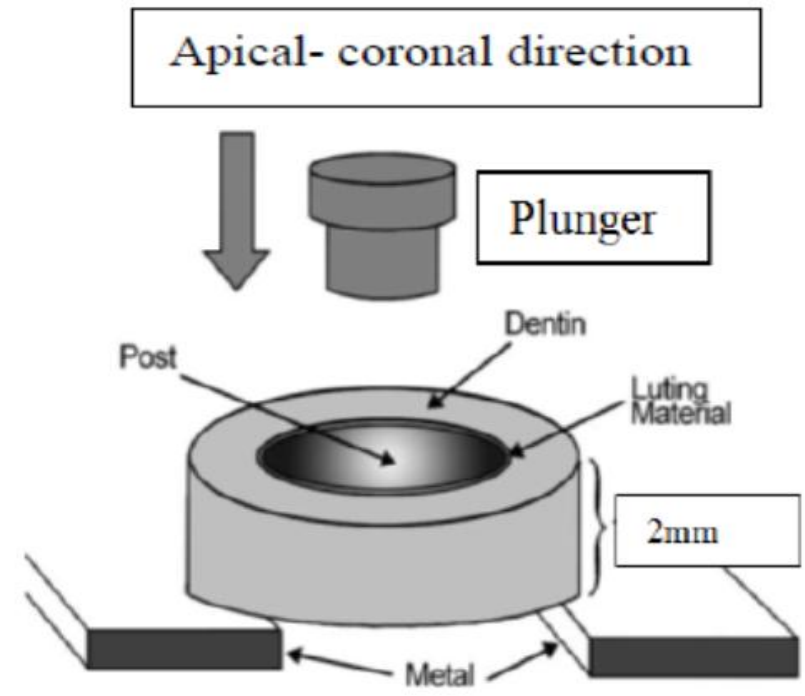

\section{Results:-}

The fabrication of teeth samples were done in Coorg Institute of Dental Sciences, Virajpet, Karnataka. The laser surface treatments for samples were done at Dr Kripa Johar's 'HariDaan Dental Clinic', Bangalore, Karnataka. The testing of samples for the tensile bond strength were done in Cauvery Polytechnic College, Gonikoppal, Karnataka. This in vitro study was carried out to evaluate and compare the tensile bond strength of two different luting agents on an endodontic fiber post after two different surface treatments. This study comprised of 60 single rooted mandibular first premolar specimens.

The teeth specimens were categorized into groups I and group II according to the cements used for luting, and further sub grouped into sub group A, B, C according to the surface treatment protocol. The groups in the study were as follows:

1. Group I: Fiber posts luted with Rely-X cement

2. Group IA: Fiber post without surface treatment luted with Rely-X cement. 
3. Group IB: Fiber post with Er:YAG laser treatment $(150 \mathrm{~mJ}$ at $10 \mathrm{~Hz}$ for $20 \mathrm{~s}$ at $100 \mu$ s pulse duration) luted with Rely-X cement.

4. Group IC: Fiber post with sandblasting $(150 \mu \mathrm{m}$ aluminium oxide was blasted for $20 \mathrm{~s}$ at an operating distance of $10 \mathrm{~mm}$ from the post) luted with Rely-X cement.

5. Group II: Fiber post luted with Calibra cement.

6. Group IIA: Fiber post without surface treatment luted with Calibra cement.

7. Group IIB: Fiber post with Er:YAG laser treatment $(150 \mathrm{~mJ}$ at $10 \mathrm{~Hz}$ for $20 \mathrm{~s}$ at $100 \mu$ s pulse duration) luted with Calibra cement.

8. Group II C: Fiber post with sandblasting $(150 \mu \mathrm{m}$ aluminium oxide was blasted for $20 \mathrm{~s}$ at an operating distance of $10 \mathrm{~mm}$ from the post) luted with Calibra cement.

The values obtained from all the groups were coded and fed in SPSS (IBM version 23) for statistical analysis. The descriptive statistics included mean and standard deviation. The independent $t$ test and one way ANOVA test followed by Post Hoc Tukey's test. The level of significance was set at 0.05 at $95 \%$ confidence interval.

The results of the study were as follows:

1. Table 1: Comparison of mean tensile bond strength of Group I among subgroups A, B and C at different segments using one way ANOVA test.

2. Table 2: Comparison of mean tensile bond strength of Group I A, B and C among different segments using Post HOC Tukey's test.

3. Table 3: Multiple comparison of mean tensile bond strength of Group I A, B and C at different segments using one way ANOVA test.

4. Table 4: Multiple comparison of mean tensile bond strength of Group I among subgroups A, B and C at different segments using post HOC Tukey's test

5. Table 5: Comparison of mean tensile bond strength of Group II among subgroups A, B and C at different segments using one way ANOVA test

6. Table 6: Comparison of mean tensile bond strength of Group II A, B and C among different segments using Post HOC Tukey's test

7. Table 7: Multiple comparison of mean tensile bond strength of Group II A, B and C at different segments using one way ANOVA test

8. Table 8: Multiple comparison of mean tensile bond strength of Group I among subgroups A, B, and C at different segments using post HOC Tukey's test

9. Table 9: Multiple comparison of mean tensile bond strength of Group I and Group II and among subgroups A, $\mathrm{B}$ and $\mathrm{C}$ at different segments using Independent $t$ test

10. Graph 1: Comparison of mean tensile bond strength of Group I and among subgroups A, B and C at different segments

11. Graph 2: Comparison of mean tensile bond strength of Group I A, B and C among different segments

12. Graph 3: Comparison of mean tensile bond strength of Group II and among subgroups A, B and C at different segments

13. Graph 4: Comparison of mean tensile bond strength of Group II A, B and C among different segments

14. Graph 5: Comparison of mean tensile bond strength among Group I A and Group II A at different segments

15. Graph 6: Comparison of tensile out bond strength among Group I B and Group II B at different segments.

16. Graph 7: Comparison of push out bond strength among Group I C and Group II C at different segments.

\section{Tensile Bond Strength}

Testing of the tensile bond strength of the cemented specimens was carried out in a Universal Testing Machine. The individual cement failure load values obtained in newton $(\mathrm{N})$ were divided by the surface area of each individual prepared tooth in $\mathrm{mm}^{2}$ to obtain the bond strength values expressed in Mega Pascal (MPa).

Table 1 shows that when comparison was made between group I (A, B, and C) at different segments using one way ANOVA test for the subgroup group I it was revealed that the mean tensile bond strength found were highly significant.

Table 2 shows that comparison of mean tensile bond strength of Group I A, B and C among different segments using Post HOC Tukey's test for group IA when comparison was made among different segments, mean tensile bond strength values were highly significant. For group IB the mean tensile bond strength values among segments were highly significant. For group IC comparison among different segments when coronal and middle segments were 
compared tensile bond strength values were not significant but when coronal segment and middle segments were compared with apical segment it was highly significant.

Table 3 shows that multiple comparison of mean tensile bond strength of Group I A, B and C at different segments using one way ANOVA test. For coronal segment comparison among subgroups A, B and C tensile bond strength was highly significant. For middle segment and apical segments comparison among subgroups A, B and C tensile bond strength were highly significant.

Table 4 shows multiple comparison of mean tensile bond strength of Group I among subgroups A, B and C at different segments using post HOC Tukey's test. Comparing coronal segments among subgroups $\mathrm{A}, \mathrm{B}$ and $\mathrm{C}$ it showed that tensile bond strength were highly significant. And when group B compared with group $\mathrm{C}$ the tensile bond strength was highly significant. Comparing middle segment among subgroups $\mathrm{A}, \mathrm{B}$ and $\mathrm{C}$ it was seen that comparing group $\mathrm{A}$ and group $\mathrm{C}$ and comparing group $\mathrm{B}$ and group $\mathrm{C}$ tensile bond strength were found to be highly significant and while comparing the group A and group B was tensile bond strength was significant.

Table 5 shows comparison of mean tensile bond strength of Group II among subgroups A, B and C at different segments using one way ANOVA test. The tensile bond strength for group II among subgroups A, B and C at different segments were highly significant.

Table 6 shows comparison of mean tensile bond strength of Group II A, B and C among different segments using Post HOC Tukey's test. For group IIA comparison among different segments the tensile bond strength values were highly significant. For group IIB while comparing the mean tensile bond strength values among segments it was highly significant. For group IIC when comparing coronal and apical segments and middle and apical segments were compared tensile bond strength values were highly significant than when comparing coronal and middle segments tensile bond strength values was significant.

Table 7 shows multiple comparison of mean tensile bond strength of Group II A, B and C at different segments using one way ANOVA test. For coronal, middle and apical segments comparison among subgroups A, B and C tensile bond strength were highly significant.

Table 8 shows multiple comparison of mean tensile bond strength of Group II among subgroups A, B, and C at different segments using post HOC Tukey's test. Comparing coronal segments among subgroups $\mathrm{A}, \mathrm{B}$ and $\mathrm{C}$ it showed that group A compared with group B tensile bond strength was significant. When group A and group C and group B and group $\mathrm{C}$ were compared tensile bond strength were highly significant.

Table 9 shows multiple comparison of mean tensile bond strength of Group I and Group II and among subgroups A, $\mathrm{B}$ and $\mathrm{C}$ at different segments using Independent $\mathrm{t}$ test. For group $\mathrm{A}, \mathrm{B}$ and $\mathrm{C}$ under coronal, middle and apical segments when group I and group II were compared, tensile bond strength were not significant.

Table 1:-Comparison of mean tensile bond strength of Group I among subgroups A, B and C at different segments using one way ANOVA test.

\begin{tabular}{|c|c|c|c|c|c|}
\hline \multicolumn{2}{|l|}{ RELY X cement } & Mean & Standard deviation & $\bar{F}$ & Significance \\
\hline \multirow[t]{3}{*}{ Control } & Coronal & 16.8370 & 1.49683 & \multirow[t]{3}{*}{50.539} & \multirow[t]{3}{*}{0.000 (H.S) } \\
\hline & Middle & 14.4670 & 1.64010 & & \\
\hline & Apical & 10.1260 & 1.39478 & & \\
\hline \multirow[t]{3}{*}{ Laser treatment } & Coronal & 19.3920 & 1.64614 & \multirow[t]{3}{*}{25.018} & \multirow[t]{3}{*}{0.000 (H.S) } \\
\hline & Middle & 16.6260 & 1.76128 & & \\
\hline & Apical & 13.4140 & 2.21834 & & \\
\hline \multirow[t]{3}{*}{ Sandblasting } & Coronal & 22.8270 & 2.05770 & \multirow[t]{3}{*}{14.766} & \multirow[t]{3}{*}{0.000 (H.S) } \\
\hline & Middle & 21.1810 & 1.57485 & & \\
\hline & Apical & 18.3380 & 1.94004 & & \\
\hline
\end{tabular}


Table 2:-Comparison of mean tensile bond strength of Group I A, B and C among different segments using Post HOC Tukey's test

\begin{tabular}{|c|c|c|c|c|c|c|c|}
\hline & & & $\begin{array}{l}\text { Mean } \\
\text { difference }\end{array}$ & $\begin{array}{l}\text { Standard } \\
\text { error }\end{array}$ & Significance & $\begin{array}{l}95 \% \\
\text { Interval }\end{array}$ & Confidence \\
\hline & & & & & & $\begin{array}{l}\text { Lower } \\
\text { bound }\end{array}$ & $\begin{array}{l}\text { Upper } \\
\text { bound }\end{array}$ \\
\hline Control & Coronal & Middle & 2.37000 & .67704 & .005 (H.S) & .6913 & 4.0487 \\
\hline & & Apical & 6.71100 & .67704 & $.000($ H.S) & 5.0323 & 8.3897 \\
\hline & Middle & Apical & 4.34100 & .67704 & $.000($ H.S) & 2.6623 & 6.0197 \\
\hline Laser & Coronal & Middle & 2.76600 & .84589 & $.008($ H.S $)$ & .6687 & 4.8633 \\
\hline treatment & & Apical & 5.97800 & .84589 & $.000($ H.S) & 3.8807 & 8.0753 \\
\hline & Middle & Apical & 3.21200 & .84589 & $.002($ H.S) & 1.1147 & 5.3093 \\
\hline Sandblasting & Coronal & Middle & 1.64600 & .83579 & .139 (N.S) & -.4263 & 3.7183 \\
\hline & & Apical & 4.48900 & .83579 & $.000($ H.S) & 2.4167 & 6.5613 \\
\hline & Middle & Apical & 2.84300 & .83579 & $.006($ H.S) & .7707 & 4.9153 \\
\hline
\end{tabular}

$\mathrm{P}<0.05$, HS-Highly Significant, NS-Non Significant

Table 3:-Multiple comparison of mean tensile bond strength of Group I A, B and C among different segments using one way ANOVA test

\begin{tabular}{|c|c|c|c|c|c|}
\hline \multicolumn{2}{|c|}{ RELY X cement } & $\begin{array}{l}\text { Mean } \\
\text { (MPa) }\end{array}$ & Standard deviation & $\mathrm{F}$ & Significance \\
\hline \multirow[t]{3}{*}{ Coronal } & Control & 16.8370 & 1.49683 & \multirow[t]{3}{*}{29.511} & \multirow[t]{3}{*}{0.000 (H.S) } \\
\hline & Laser treatment & 19.3920 & 1.64614 & & \\
\hline & Sandblasting & 22.8270 & 2.05770 & & \\
\hline \multirow[t]{3}{*}{ Middle } & Control & 14.4670 & 1.64010 & \multirow[t]{3}{*}{42.605} & \multirow[t]{3}{*}{0.000 (H.S) } \\
\hline & Laser treatment & 16.6260 & 1.76128 & & \\
\hline & Sandblasting & 21.1810 & 1.57485 & & \\
\hline \multirow[t]{3}{*}{ Apical } & Control & 10.1260 & 1.39478 & \multirow[t]{3}{*}{48.209} & \multirow[t]{3}{*}{0.000 (H.S) } \\
\hline & Laser treatment & 13.4140 & 2.21834 & & \\
\hline & Sandblasting & 18.3380 & 1.94004 & & \\
\hline
\end{tabular}

Table 4:-Multiple comparison of mean tensile bond strength of Group I among subgroups A, B and C at different segments using post HOC Tukey's test

\begin{tabular}{|c|c|c|c|c|c|c|c|}
\hline & & & \multirow[t]{2}{*}{$\begin{array}{l}\text { Mean } \\
\text { difference }\end{array}$} & \multirow[t]{2}{*}{$\begin{array}{l}\text { Standard } \\
\text { error }\end{array}$} & \multirow[t]{2}{*}{ Significance } & \multicolumn{2}{|c|}{$\begin{array}{ll}95 \% & \text { Confidence } \\
\text { Interval } & \\
\end{array}$} \\
\hline & & & & & & $\begin{array}{l}\text { Lower } \\
\text { bound }\end{array}$ & $\begin{array}{l}\text { Upper } \\
\text { bound }\end{array}$ \\
\hline \multirow[t]{3}{*}{ Coronal } & \multirow[t]{2}{*}{ Control } & Laser treatment & -2.55500 & .78249 & $.008($ H.S) & -4.4951 & -.6149 \\
\hline & & Sandblasting & -5.99000 & .78249 & $.000($ H.S) & -7.9301 & -4.0499 \\
\hline & $\begin{array}{l}\text { Laser } \\
\text { treatment }\end{array}$ & Sandblasting & -3.43500 & .78249 & $.000($ H.S) & -5.3751 & -1.4949 \\
\hline \multirow[t]{3}{*}{ Middle } & \multirow[t]{2}{*}{ Control } & Laser treatment & -2.15900 & .74262 & (019(S) & -4.0003 & -.3177 \\
\hline & & Sandblasting & -6.71400 & .74262 & $.000($ H.S) & -8.5553 & -4.8727 \\
\hline & $\begin{array}{l}\text { Laser } \\
\text { treatment }\end{array}$ & Sandblasting & -4.55500 & .74262 & $.000($ H.S) & -6.3963 & -2.7137 \\
\hline \multirow[t]{3}{*}{ Apical } & \multirow[t]{2}{*}{ Control } & Laser treatment & -3.28800 & .84183 & $.002($ H.S) & -5.3753 & -1.2007 \\
\hline & & Sandblasting & -8.21200 & .84183 & $.000($ H.S) & -10.2993 & -6.1247 \\
\hline & $\begin{array}{l}\text { Laser } \\
\text { treatment }\end{array}$ & Sandblasting & -4.92400 & .84183 & $.000($ H.S) & -7.0113 & -2.8367 \\
\hline
\end{tabular}


Table 5:-Comparison of mean tensile bond strength of Group II among subgroups A, B and C at different segments using one way ANOVA test

\begin{tabular}{|c|c|c|c|c|c|}
\hline \multicolumn{2}{|l|}{ Calibra cement } & $\begin{array}{l}\text { Mean } \\
(\mathrm{MPa})\end{array}$ & Standard deviation & $\bar{F}$ & Significance \\
\hline \multirow[t]{3}{*}{ Control } & Coronal & 17.6790 & 1.83161 & \multirow[t]{3}{*}{37.600} & \multirow[t]{3}{*}{0.000 (H.S) } \\
\hline & Middle & 14.8630 & 2.23999 & & \\
\hline & Apical & 11.0480 & .68165 & & \\
\hline \multirow[t]{3}{*}{ Laser treatment } & Coronal & 19.8650 & 1.15160 & \multirow[t]{3}{*}{47.103} & \multirow[t]{3}{*}{0.000 (H.S) } \\
\hline & Middle & 16.5470 & 1.38659 & & \\
\hline & Apical & 14.3710 & 1.27534 & & \\
\hline \multirow[t]{3}{*}{ Sandblasting } & Coronal & 24.1430 & 1.69708 & \multirow[t]{3}{*}{21.937} & \multirow[t]{3}{*}{0.000 (H.S) } \\
\hline & Middle & 22.1020 & 1.83891 & & \\
\hline & Apical & 19.1290 & 1.55963 & & \\
\hline
\end{tabular}

Table 6:-Comparison of mean tensile bond strength of Group II A, B and C among different segments using Post HOC Tukey's test

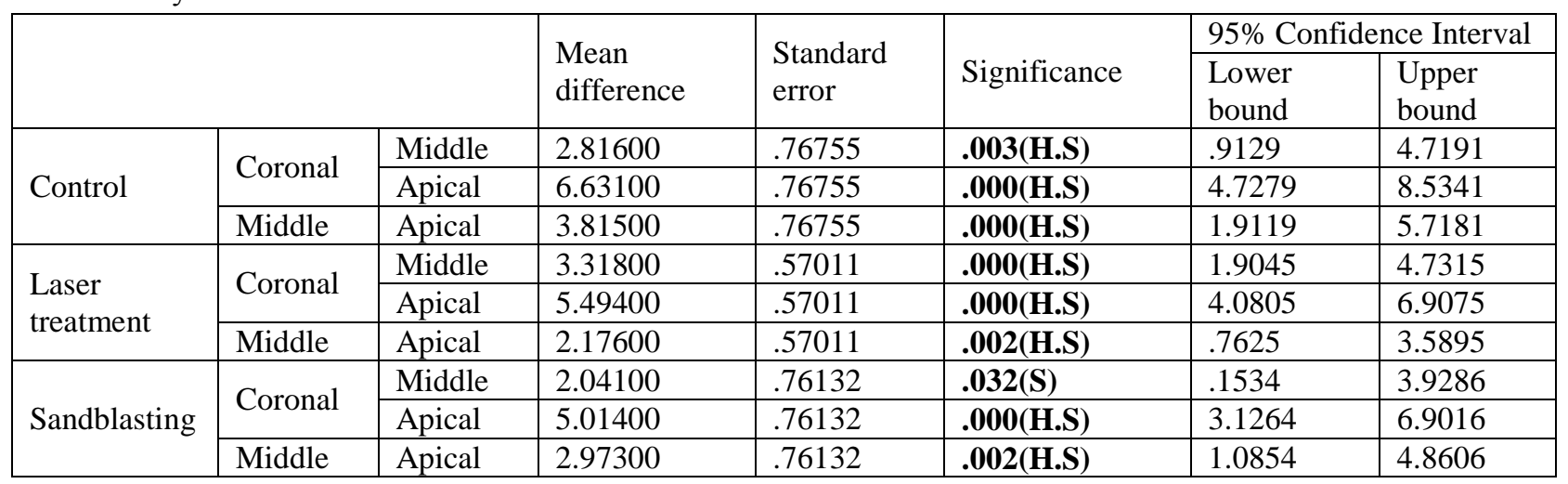

Table 7:-Multiple comparison of mean tensile bond strength of Group II A, B and C among different segments using Post HOC Tukey's test

\begin{tabular}{|c|c|c|c|c|c|}
\hline \multicolumn{2}{|c|}{ Calibra cement } & \multirow{2}{*}{$\begin{array}{l}\begin{array}{l}\text { Mean } \\
(\mathrm{MPa})\end{array} \\
17.6790\end{array}$} & \multirow{2}{*}{$\begin{array}{l}\text { Standard deviation } \\
1.83161\end{array}$} & \multirow{2}{*}{$\begin{array}{l}\mathrm{F} \\
42.893\end{array}$} & \multirow{2}{*}{$\begin{array}{l}\text { Sig. } \\
\mathbf{0 . 0 0 0} \text { (H.S) }\end{array}$} \\
\hline \multirow{3}{*}{ Coronal } & Control & & & & \\
\hline & Laser treatment & 19.8650 & 1.15160 & & \\
\hline & Sandblasting & 24.1430 & 1.69708 & & \\
\hline \multirow[t]{3}{*}{ Middle } & Control & 14.8630 & 2.23999 & \multirow[t]{3}{*}{41.706} & \multirow[t]{3}{*}{0.000 (H.S) } \\
\hline & Laser treatment & 16.5470 & 1.38659 & & \\
\hline & Sandblasting & 22.1020 & 1.83891 & & \\
\hline \multirow[t]{3}{*}{ Apical } & Control & 11.0480 & .68165 & \multirow[t]{3}{*}{109.408} & \multirow[t]{3}{*}{ 0.000 (H.S) } \\
\hline & Laser treatment & 14.3710 & 1.27534 & & \\
\hline & Sandblasting & 19.1290 & 1.55963 & & \\
\hline
\end{tabular}

Table 8:-Multiple comparison of mean tensile bond strength of Group II among subgroups A, B, and C at different segments using post HOC Tukey's test

\begin{tabular}{|l|l|l|l|l|l|l|l|}
\hline \multicolumn{2}{|c|}{} & \multirow{2}{*}{$\begin{array}{l}\text { Mean } \\
\text { difference }\end{array}$} & $\begin{array}{l}\text { Standard } \\
\text { error }\end{array}$ & Significance & $\begin{array}{l}\text { 95\% } \\
\text { Interval }\end{array}$ \\
\cline { 4 - 8 } & & & & $\begin{array}{l}\text { Lower } \\
\text { bound }\end{array}$ & $\begin{array}{l}\text { Upper } \\
\text { bound }\end{array}$ \\
\hline \multirow{2}{*}{ Coronal } & Control & Laser treatment & -2.18600 & .70998 & $\mathbf{. 0 1 3}(\mathbf{S})$ & -3.9463 & -.4257 \\
\cline { 2 - 8 } & Sandblasting & -6.46400 & .70998 & $\mathbf{. 0 0 0}($ H.S) & -8.2243 & -4.7037 \\
\cline { 2 - 8 } & $\begin{array}{l}\text { Laser } \\
\text { treatment }\end{array}$ & Sandblasting & -4.27800 & .70998 & $\mathbf{. 0 0 0}($ H.S) & -6.0383 & -2.5177 \\
\hline
\end{tabular}




\begin{tabular}{|l|l|l|l|l|l|l|l|}
\hline Middle & Control & Laser treatment & -1.68400 & .82953 & .124 (N.S) & -3.7407 & .3727 \\
\cline { 2 - 8 } & & Sandblasting & -7.23900 & .82953 & $\mathbf{. 0 0 0}($ H.S) & -9.2957 & -5.1823 \\
\cline { 2 - 7 } & $\begin{array}{l}\text { Laser } \\
\text { treatment }\end{array}$ & Sandblasting & -5.55500 & .82953 & $\mathbf{. 0 0 0 ( H . S )}$ & -7.6117 & -3.4983 \\
\hline \multirow{2}{*}{ Apical } & Control & Laser treatment & -3.32300 & .54916 & $\mathbf{. 0 0 0 ( H . S )}$ & -4.6846 & -1.9614 \\
\cline { 2 - 8 } & Sandblasting & -8.08100 & .54916 & $\mathbf{. 0 0 0 ( H . S )}$ & -9.4426 & -6.7194 \\
\cline { 2 - 8 } & $\begin{array}{l}\text { Laser } \\
\text { treatment }\end{array}$ & Sandblasting & -4.75800 & .54916 & $\mathbf{. 0 0 0}$ (H.S) & -6.1196 & -3.3964 \\
\hline
\end{tabular}

Table 9:-Multiple comparison of mean tensile bond strength of Group I and Group II and among subgroups A, B and $\mathrm{C}$ at different segments using Independent $\mathrm{t}$ test

\begin{tabular}{|c|c|c|c|c|c|c|}
\hline & & & $\begin{array}{l}\text { Mean } \\
(\mathrm{MPa})\end{array}$ & $\begin{array}{l}\text { Standard } \\
\text { deviation }\end{array}$ & $\mathrm{T}$ & Significance \\
\hline \multirow[t]{6}{*}{ Control } & \multirow[t]{2}{*}{ Coronal } & Rely X & 16.8370 & 1.49683 & \multirow[t]{2}{*}{-1.126} & \multirow[t]{2}{*}{0.275 (N.S) } \\
\hline & & Calibra & 17.6790 & 1.83161 & & \\
\hline & \multirow[t]{2}{*}{ Middle } & Rely X & 14.4670 & 1.64010 & \multirow[t]{2}{*}{-0.451} & \multirow[t]{2}{*}{0.102 (N.S) } \\
\hline & & Calibra & 14.8630 & 2.23999 & & \\
\hline & \multirow[t]{2}{*}{ Apical } & Rely X & 10.1260 & 1.39478 & \multirow[t]{2}{*}{-1.878} & \multirow[t]{2}{*}{$0.102($ N.S) } \\
\hline & & Calibra & 11.0480 & .68165 & & \\
\hline \multirow[t]{6}{*}{ Laser treated } & \multirow[t]{2}{*}{ Coronal } & Rely X & 19.3920 & 1.64614 & \multirow[t]{2}{*}{-0.745} & \multirow[t]{2}{*}{$0.150($ N.S) } \\
\hline & & Calibra & 19.8650 & 1.15160 & & \\
\hline & \multirow[t]{2}{*}{ Middle } & Rely X & 16.6260 & 1.76128 & \multirow[t]{2}{*}{0.111} & \multirow[t]{2}{*}{0.405 (N.S) } \\
\hline & & Calibra & 16.5470 & 1.38659 & & \\
\hline & \multirow[t]{2}{*}{ Apical } & Rely X & 13.4140 & 2.21834 & \multirow[t]{2}{*}{-1.183} & \multirow[t]{2}{*}{$0.071($ N.S) } \\
\hline & & Calibra & 14.3710 & 1.27534 & & \\
\hline \multirow[t]{6}{*}{ Sandblasting } & \multirow[t]{2}{*}{ Coronal } & Rely X & 22.8270 & 2.05770 & \multirow[t]{2}{*}{-1.560} & \multirow[t]{2}{*}{$0.534(\mathrm{~N} . \mathrm{S})$} \\
\hline & & Calibra & 24.1430 & 1.69708 & & \\
\hline & \multirow[t]{2}{*}{ Middle } & Rely X & 21.1810 & 1.57485 & \multirow[t]{2}{*}{-1.203} & \multirow[t]{2}{*}{$0.211(\mathrm{~N} . \mathrm{S})$} \\
\hline & & Calibra & 22.1020 & 1.83891 & & \\
\hline & \multirow[t]{2}{*}{ Apical } & Rely X & 18.3380 & 1.94004 & \multirow[t]{2}{*}{-1.005} & \multirow[t]{2}{*}{ 0.739(N.S) } \\
\hline & & Calibra & 19.1290 & 1.55963 & & \\
\hline
\end{tabular}

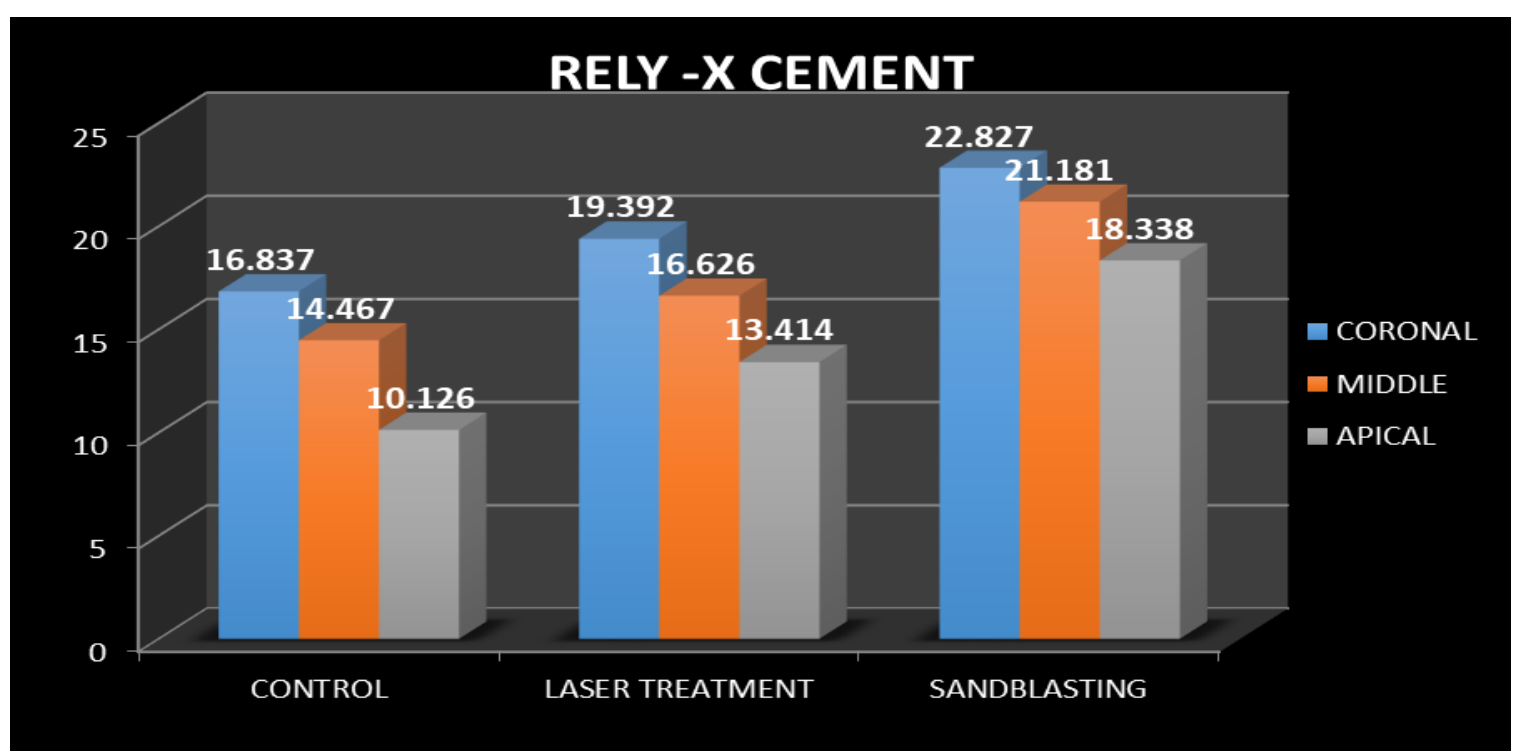

Graph 1:-Comparison of mean tensile bond strength of Group I and among subgroups A, B and C at different segments (all values are I Mega Pascal - MPa). 


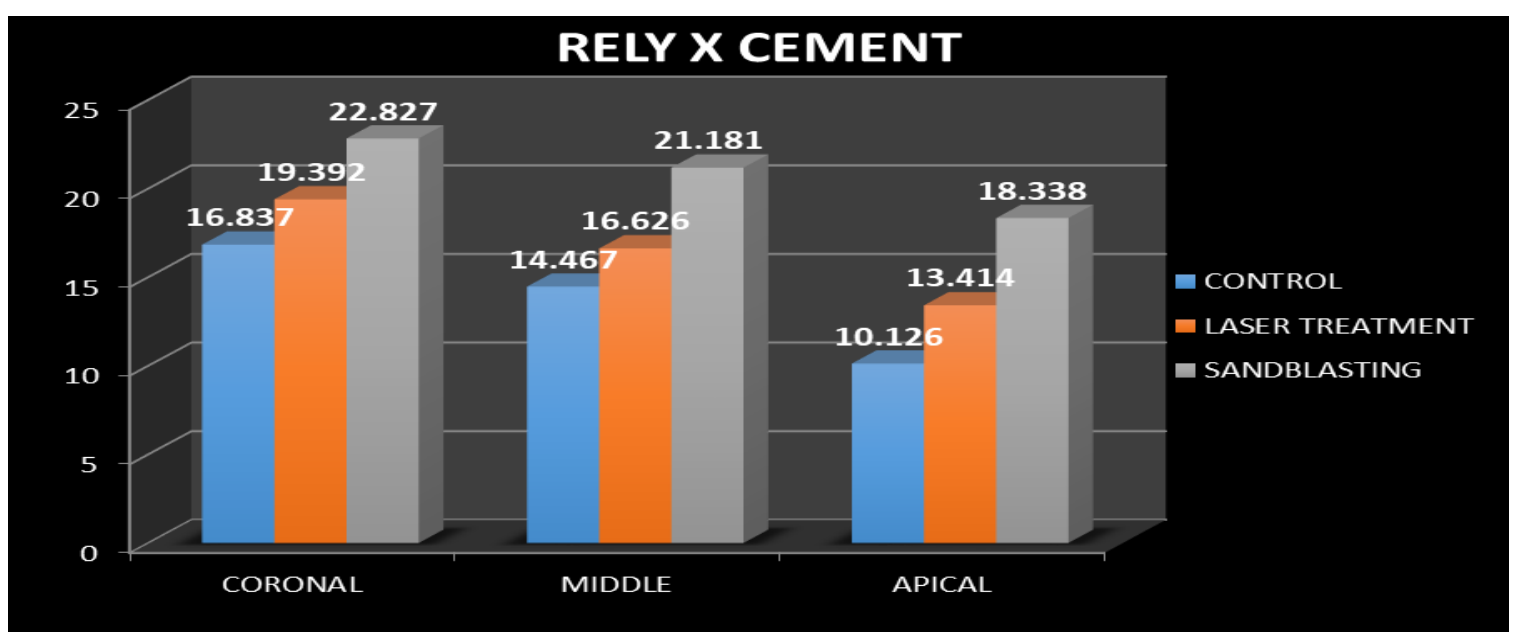

Graph 2:-Comparison of mean tensile bond strength of Group I A, B and C among different segments (all values are I Mega Pascal - MPa).

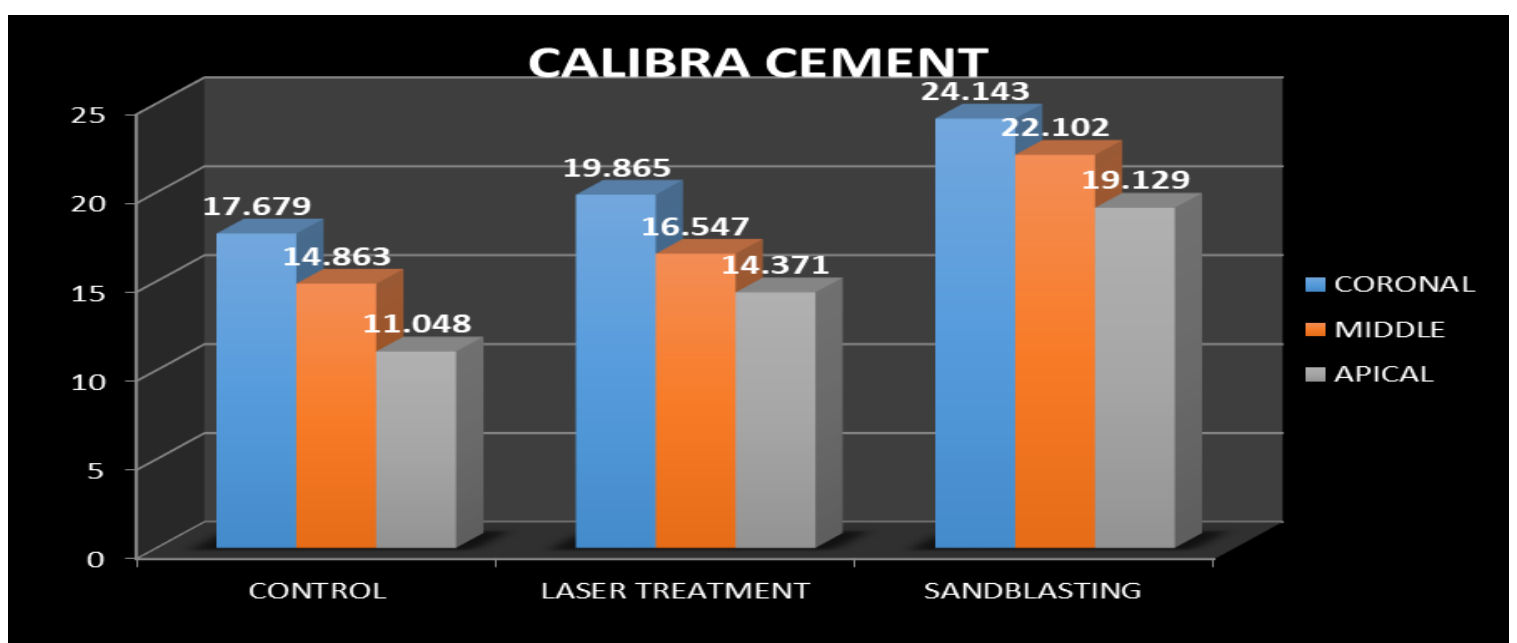

Graph 3:-Comparison of mean tensile bond strength of Group II and among subgroups A, B and C at different segments (all values are I Mega Pascal - MPa).

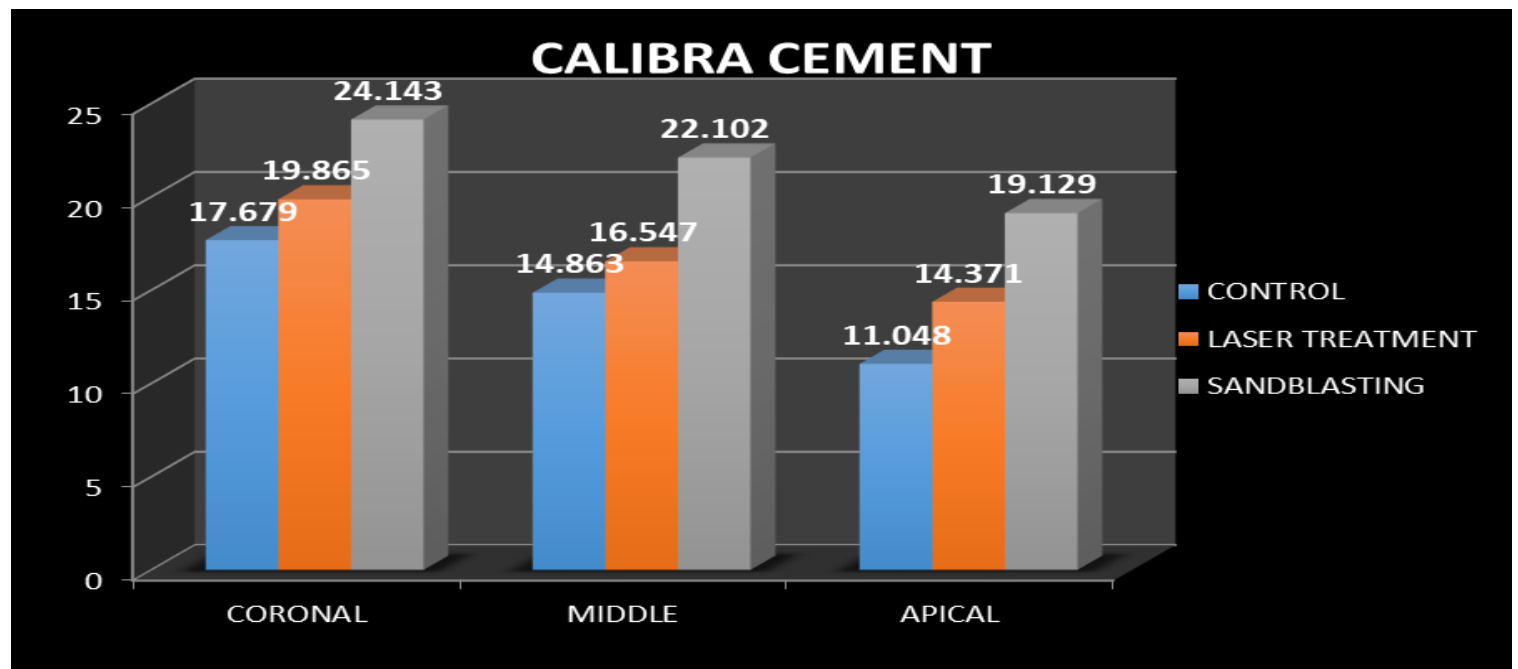

Graph 4:-Comparison of mean tensile bond strength of Group II A, B and C among different segments (all values are I Mega Pascal - MPa). 


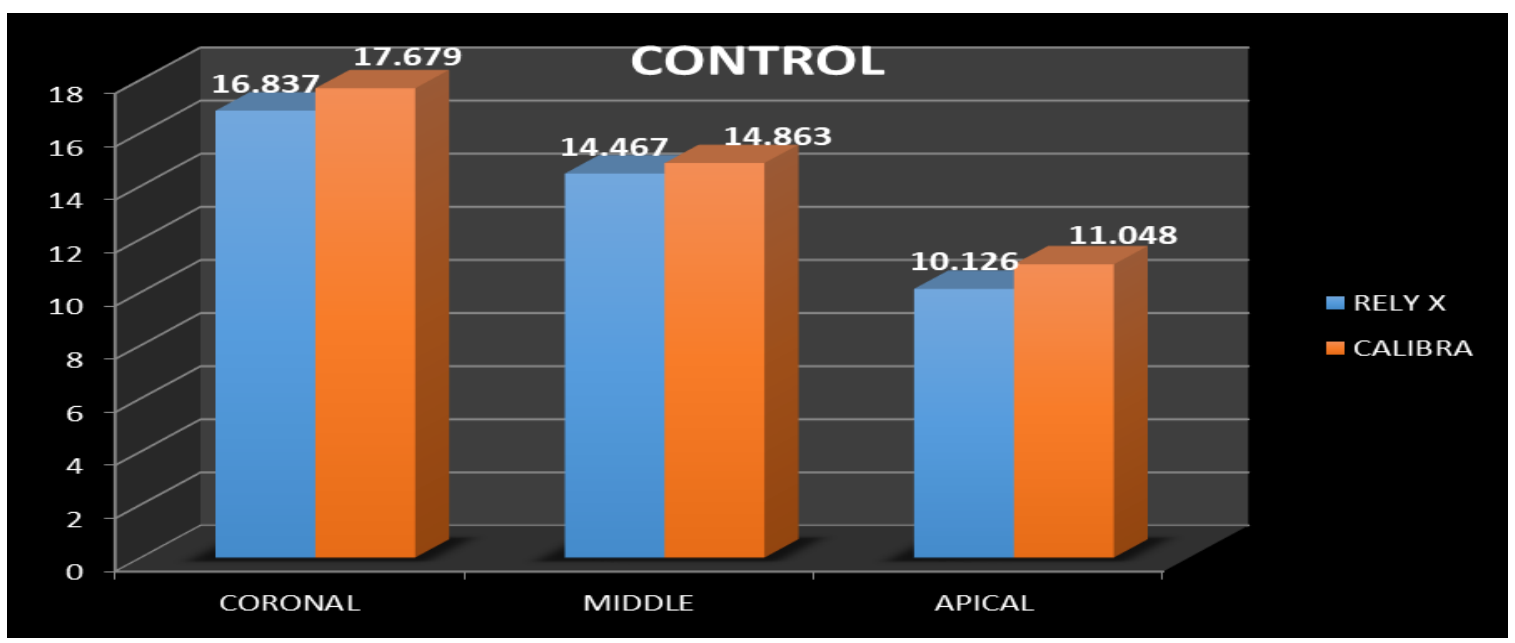

Graph 5:-Comparison of mean tensile bond strength among Group I A and Group II A at different segments (all values are I Mega Pascal - MPa).

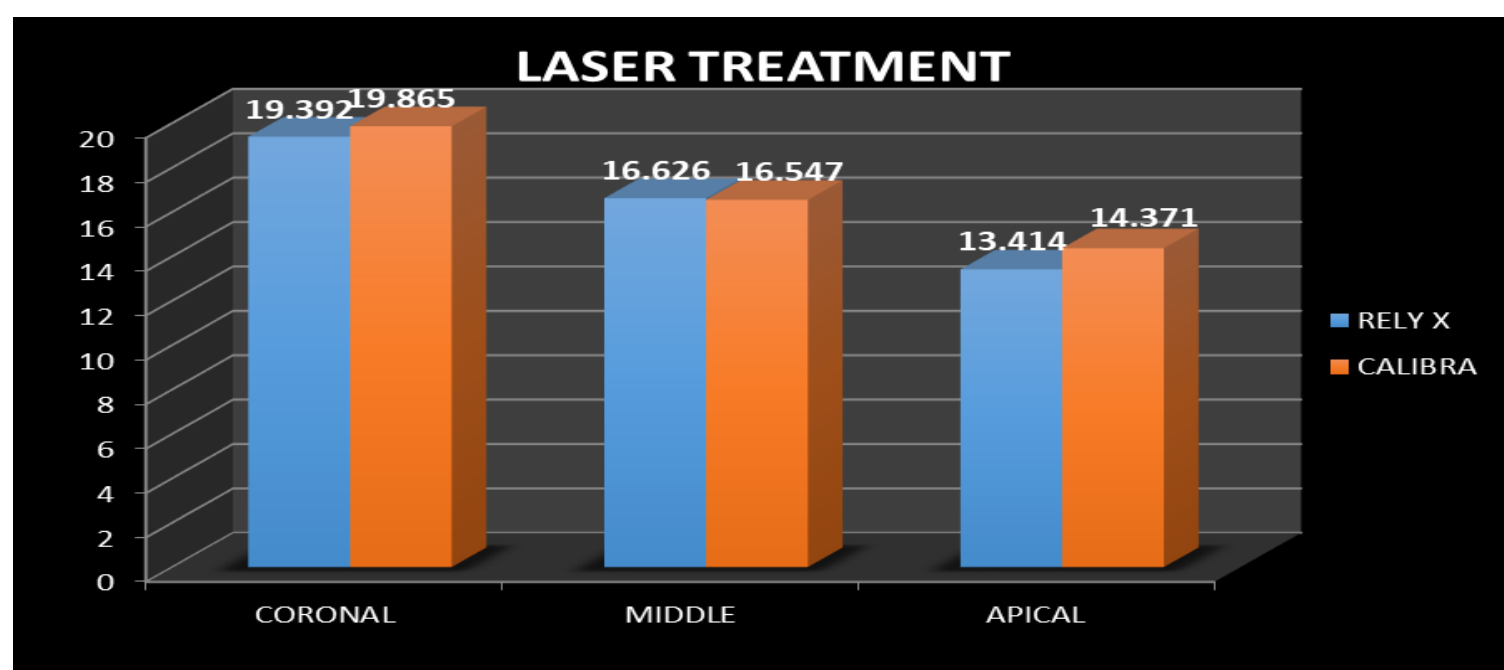

Graph 6:-Comparison of push out bond strength among Group I B and Group II B at different segments (all values are I Mega Pascal - MPa).

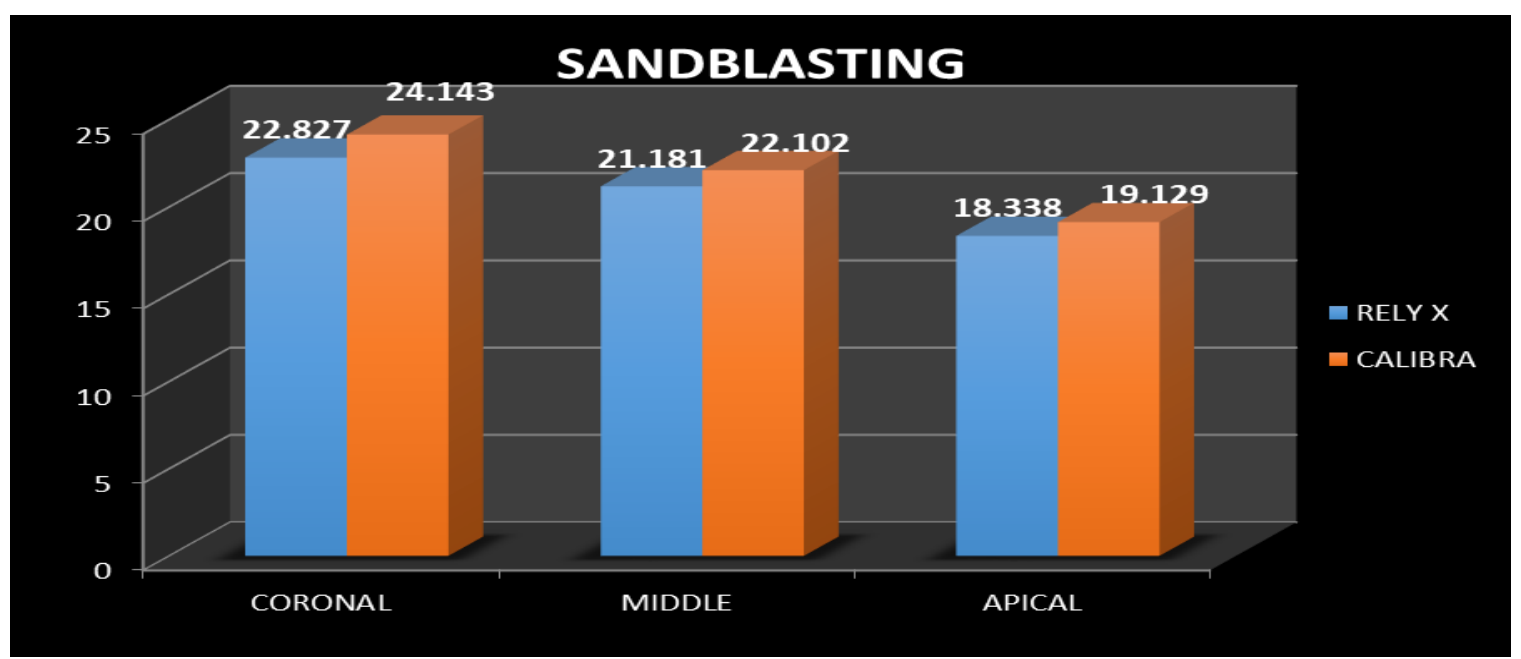

Graph 7:-Comparison of push out bond strength among Group I C and Group II C at different segments (all values are I Mega Pascal - MPa). 


\section{Discussion:-}

Endodontically treated teeth are known to present a higher risk of biomechanical failure than vital teeth. Posts are generally indicated to restore missing tooth structure and pulpless teeth. Placement of a post should be considered only when the remaining cervical tooth tissue can no longer provide adequate support and retention for a restoration. ${ }^{23}$ Posts are widely used for restoring endodontically treated teeth with insufficient coronal tooth structure to retain a core for the definitive restoration. ${ }^{2}$

When using posts, factors such as the length, design, and material of the post should be considered. ${ }^{23}$ Metal posts were reported to have less retention, cause serious types of root fractures, compromise esthetics and are susceptible to corrosion. ${ }^{1}$ The rigid metal posts can defy to the lateral forces without deformation because of the homogeneous structure. However, this rigidity causes root fractures according to the stress distribution. ${ }^{4}$ There has been rapidly increasing development and use of these fiber -reinfiorced composite root canal posts over the last 10 years. As alternatives, fiber-reinforced composite (FRC) posts were developed with intensive research interest. Many investigators have suggested that these materials have the advantage of reducing the risk of root fracture thanks to their modulus of elasticity (16-40 GPa) being comparable with that of composite resins (5.7-25 GPa) and dentin $(18.6 \mathrm{GPa}){ }^{1}$

Glass fiber-reinforced resin post systems were introduced in 1992. These posts are composed of unidirectional glass fibers embedded in a resin matrix. Matrix polymers are commonly epoxy polymers with a high degree of monomer conversion and a highly cross-linked structure. An advantage of glass fibers is that they distribute stress over a broad surface area, increasing the load threshold at which the post begins to show evidence of micro-fractures. Consequently, fiber-reinforced posts are reported to reduce the risk of tooth fractures and display higher survival rates than teeth restored with rigid zirconia posts. ${ }^{23}$ Furya $U$ et al evaluated the fracture strength and stress distributions of pulpless premolars restored with fiber posts. The study examined the effect of glass fiber posts on increasing the fracture resistance of endodontically treated teeth. The result of the study showed that teeth with glass fiber post(s) showed significantly higher fracture loads compared with those without posts. ${ }^{8}$ Hence glass fiber post are used in this study.

Glass-fiber posts are composed of glass fibers, inorganic filler, and a resin matrix. They are usually luted with a resin cement to increase their retention and improve the mechanical performance of the restored teeth. In many studies, different surface treatments were tested to investigate the bond strength between a composite resin matrix and resin cement. ${ }^{2}$

The polymer matrix in the structure of FRC post is unable to react with the monomers of resin cements. On the basis of this fact, it has been suggested to apply surface preparation processes that selectively react with the epoxy resin matrix of the FRC post in order to roughen the post surface and expose the glass fibers. These procedures allow the micro-mechanical interlocking of the adhesive/cement with the post. ${ }^{4}$

Non-treated fiber posts have a relatively smooth surface area that limits mechanical interlocking between the post surface and resin cement, and purely adhesive failure modes are commonly recorded at the post/composite interfaces. $^{24}$ A number of studies particularly focused on the possibility of improving adhesion at the fiber postcomposite interface through various treatments of the post surface. In an attempt to maximize resin bonding to FRC posts, several surface treatments were recently suggested. These procedures can be divided into three categories: (1) silanization and/or adhesive application, (2) acid etching, sandblasting, and silica coating, and (3) alternative etching techniques (i.e., treatments that combine both a micromechanical and chemical component). ${ }^{5}$ Sandblasting of the dental restorations is often used to clean the materials, to increase the surface area, and to ensure the micro-retentive areas. $^{4}$ It is well accepted that sandblasting with alumina particles results in increased surface roughness and surface area. The mechanical action of blasting probably determines the removal of the superficial layer of the resinous matrix, creating microretentive spaces on the post surface. ${ }^{19}$

Recently, lasers have become popular in dentistry. Laser technology is being developed very quickly and is beginning to be used in the different fields of dentistry. A new type of laser called Er:YAG (erbium-doped yttrium aluminium garnet laser) emitted in the wavelength of 2,940 nm matches exactly the maximal absorption in water. Also, well absorbed in hydroxyapatite, this laser seems to have been made for the effective removal of dentin and enamel with only minor side-effects such as thermal damage. The potential of Er:YAG lasers for the ablation of hard tissue in dentistry was demonstrated already in 1989. Many of the technological advances have been directed at the 
use of lasers in clinical settings as an alternative to the acid etching of dental materials or teeth for improving bond strength.

Due to improvements in lasers used in dentistry, Erbium: Yttrium- Aluminum- Garnet (Er:YAG) laser treatment is considered an alternative method to other surface treatment methods because of its optical penetration depth. ${ }^{19}$ Hence in this study sandblasting and Er:YAG laser surface treatments were used on glass fiber post .

Selecting an appropriate adhesive and luting procedure for bonding posts to root dentin is another challenge. Sealing is expected to be strong due to recent improvements in the sealing ability of adhesive resin luting agents. In a recent investigation, carbon fiber post and core foundations cemented with dentin bonding and resin luting agents showed less microleakage than those luted with glass-ionomer and zinc-phosphate cements. ${ }^{23}$ The success of the root dentin adhesive restorative technique is directly associated with the hybridization quality produced by the adhesive system infiltration into the demineralized dentin substrate. Innumerable number of post cement materials are available in the market claiming various levels of efficiency. The credibility of these cements when clubbed with surface treated posts though has not been analyzed in detail.

The selection of the composite plays an important role, not only for the clinical success of a post/core restoration but also for the quality of the post/core interface, where different materials are in close contact. Resin-based adhesive luting materials are widely used for the fixation of posts, and currently all the resin cements are based upon the use of either an etch-and-rinse or of a self-etch adhesive, along with a low-viscosity resin composite. ${ }^{9}$ The use of resin cement has been found to significantly increase retention of posts and fracture resistance of the tooth compared with other cements. Some studies have reported that fiber-reinforced resin posts provided lower retention when compared with metal posts. ${ }^{2}$ Various luting agents have been proposed for bonding fiber-reinforced composite (FRC) posts to root canal dentin used with self-etching or etch-and-rinse adhesive systems. In recent years, new resin cement formulas have been developed that have a self-adhesive capacity. These cements have the advantage of not requiring any dentin pretreatment. ${ }^{5}$ Hence resin luting cements ( RelyX and Calibra ) were selected for the study.

In this invitro study evaluation and comparison of the tensile bond strength of two different luting agents on an endodontic fiber post after two different surface treatments was conducted.

To assess the bond strength between fiber posts and the root canal dentin, conventional shear and tensile tests, microshear, microtensile, and pull-out and push-out tests have been used. It is suggested that the bond strength is better obtained by the push-out test than with the conventional shear test because the fracture occurs parallel to the dentin bonding interface in the push-out test, which makes it a true shear test. As premature failures occur during specimen preparation and because of the large data distribution observed in microtensile tests, the push-out test is considered more reliable. ${ }^{15}$ Therefore this invitro study was aimed at evaluating and comparing the effect of sand blasting and laser surface treatment on the retention of endodontic fiber posts luted with two commonly used luting agents Rely-X U 200 and Calibra cements (both are self-adhesive resin cements).

\section{The objectives of this study were}

1. To compare the interfacial bond strengths of different resin based luting cements that are used for bonding of fiber posts to intact root canals using a push out test.

2. To evaluate and compare the tensile bond strength of fiber posts with and without sandblasting luted with Rely$\mathrm{X}$ cement and with Calibra cement.

3. To evaluate and compare the tensile bond strength of fiber posts with and without laser treatment luted with Rely-X cement and with Calibra cement.

4. To evaluate and compare the tensile bond strength of sandblasted fiber posts to the laser treated fiber posts luted with Rely-X cement and with Calibra cement.

In this study sixty mandibular first premolars were selected and cut perpendicular to the long axis after marking 14 $\mathrm{mm}$ from the apex of the tooth towards the incisal surface. Root canals were prepared using step-back technique and obturated with gutta-percha using lateral condensation. Post space preparation was done using Peeso Reamers. The roots were divided into 2 experimental groups based on the luting cements and further divided into 3 subgroups according to surface treatments used $(\mathrm{n}=10)$. (10 control group, 10 sandblasted group, 10 laser treated group). Resin luting agents (Rely X U200 \& Calibra) were mixed according to manufacturer's instruction and then placed in the post spaces and the posts were cemented to the prepared specimens. Each root was cut horizontally, and three 2- 
mm-thick root segments ( 1 apical, 1 middle, and 1 cervical) were prepared. The specimens were later stored in saline for $24 \mathrm{hrs}$ at $37^{\circ} \mathrm{C}$. Using a push-out test, the bond strength between post and dentin was measured using a universal testing machine. The individual cement failure load values obtained in Newton (N) were divided by the surface area of each individual prepared tooth in $\mathrm{mm}^{2}$ to obtain the bond strength values expressed in Mega Pascal $(\mathrm{MPa})$.

The values obtained from all groups were coded and fed in SPSS (IBM version 23) for statistical analysis. The descriptive statistics included mean and standard deviation. The independent $t$ test and one way ANOVA test followed by Post Hoc Tukey's test. The level of significance was set at 0.05 at $95 \%$ confidence interval were applied.

The results obtained from the present study revealed that when comparison was made between group I (A, B, and C) at different segments using one way ANOVA test for the subgroups group I it was revealed that the mean tensile bond strength found were highly significant and it wasn't revealed which segments showed better bond strength. So to find this, Post Hoc Tukey test was applied further then on evalution of tensile bond strength of 2 different luting agents on an endodontic fiber post after two different surface treatments at different segments, coronal segment showed the highest bond strength for both the groups than the middle and apical segments at $p$ value $=0.00$

However, some aspects related to intraradicular dentin remain uncertain, as some failures have been clinically observed. Different root regions show different distributions and densities of dentin tubules. The densities and the number of dentin tubules decreased significantly from the coronal to apical root regions. According to Gwinnett these tags contribute about $30 \%$ to the total bond strength. These findings seem to suggest that if there are fewer tubules per square millimeter in the apical region, the bond strength will be lower since there will be less resin tag formation. The better performance in the coronal region is also attributed to the fact that this is the most accessible part of the canal space, making it easier to thoroughly apply the adhesive agents. ${ }^{1}$

In addition, the apical areas of post preparation in the root canal pose additional difficulties with regard to the insertion and photo-activation of adhesive restorative systems. The difficulty in obtaining direct light irradiation in apical regions is likely to be the main reason for the lower bond effectiveness in this region .There is a significant reduction in the quantity of light transmitted into the root canal as the depth increases, and this has been shown to reach levels insufficient for achieving polymerization, especially in the apical third. ${ }^{1}$

Other factors possibly interfering with the development of high bond strengths to root dentin are the nonuniform adaptation of the bonding material or its incomplete polymerization, both related to the difficult access of post space walls during handling. These factors may account for the lower bond strengths achieved by the adhesive cements in the middle and apical root segments. ${ }^{23}$ Hence, this study showed similar results where coronal segments had highly significant tensile bond strength value than compared with middle and apical segments for both the cements (i.e group I \& II).

On multiple comparison of mean tensile bond strength of Group I (Rely X cement) among subgroups (A,B and C) at different segments using one way ANOVA test, for each segment (coronal, middle \& apical) comparison among subgroups A, B and C tensile bond strength was highly significant $(\mathrm{P}=0.00)$ that is when control group compared with laser and sandblasting groups it was shown that bond strength were improved on the surface treated groups. But among the surface treatments sandblasting showed better bond strength values than laser surface treatment which was highly significant $(\mathrm{p}=0.00)$.

This result is consistent with previous studies that reported that airborne-particle abrasion with aluminum oxide particles increased the surface area and enhanced the mechanical interlocking between the cement and the roughened surface of a post.

Choi et al studied the effect of surface treatment of fiber-reinforced posts on adhesion of a resin-based luting agent. In this study the surface treatments used were following surface treatment procedures: no treatment (NS) (control), silanization (SA) (Monobond-S) , airborne-particle abrasion (AB) (Airsonic Alu-Oxyd), or silanization subsequent to airborne-particle abrasion (AB plus SA). They had also used aluminum oxide (particle size: $50 \mu \mathrm{m}$ ) at Airborneparticle abrasion from distance of $1 \mathrm{~cm}$ at $2.8 \mathrm{bar}(0.28 \mathrm{MPa})$ for $5 \mathrm{~s}$ and they had also explained that since the post surface was smaller than $1 \mathrm{~cm}^{2}$ (for which airborne-particle abrasion for 15 seconds was recommended). 
This approach has been recently reported to be effective, minimizing dimensional changes with the fiber posts. For these reasons, the post surfaces were airborne-particle abraded with 50- $\mu \mathrm{m}$ aluminum oxide particles at 2.8-bar $(0.28$ $\mathrm{MPa}$ ) pressure for only 5 seconds (because of the small bonding surface) from a distance of $10 \mathrm{~mm}$ in the current study the $\mathrm{Al}_{2} \mathrm{O}_{3}$ used was $150 \mu \mathrm{m}$ particle size was blasted for 20 secs at an operating distance of $10 \mathrm{~mm}$ from the post. According to the previous study it was proved that the reduction in airbone -particle-abration time can reduce the effect it was shown that in present study sandblasting had comparatively more effect since the particle size and abration time are more. The highest bond strength was recorded in the airborne-particle-abraded group with no additional surface treatment ${ }^{16}$.

Any surface treatment carried out on the post is likely to cause changes in the mechanical properties of the posts. Although comparative studies showing the advantages of various types of surface pretreatment methods on fiber post exist there has been no consensus in the literature regarding the best surface pretreatment method for optimum bonding. ${ }^{21}$

Hence within the limitation of the present study it is revealed that among the surface treatments applied over glass fiber post sandblasting was more effective had highly significant values of tensile bond strength compared with laser surface treatment.

For group II (Calibra cement) had also shown similar results like group I when comparison of mean tensile bond strength was done among the subgroups at different segments using one way ANOVA test. Which was proved to be similar to earlier studies.

When comparing the mean tensile bond strength of group I when comparison is done among the subgroups (A, B, C) at different segments, it was found that among sandblasting group when coronal and middle segments were compared the tensile bond strength values were found to be high but not significant. Because the densities and the number of dentin tubules decreased significantly from the coronal to apical root regions. According to Gwinnett these tags contribute about $30 \%$ to the total bond strength. These findings seem to suggest that if there are fewer tubules per square millimeter in the apical region, the bond strength will be lower since there will be less resin tag formation. ${ }^{1}$ So the values obtained for coronal and middle segments appeared to be similar hence the values obtained showed not significant.

When multiple comparison of mean tensile bond strength of group I among subgroups at different segments using Post Hoc Tukeys test among middle segment when control group is compared with laser group tensile bond strength value was found to be high but not that significant. Because untreated fiber posts have a relatively smooth surface area that limits mechanical interlocking between the post surface and resin cement, and purely adhesive failure modes are commonly recorded at the post/composite interfaces. ${ }^{24}$

When mean tensile bond strength of group II is compared among subgroups and between the different segments using Post Hoc Tukeys test it was seen that among group $\mathrm{C}$ when coronal and middle segments were compared the tensile bond strength value were high but significant than tensile bond strength values compared among coronal with apical and middle and apical. Hence it may be due to the similar size of dentinal tubueles as explained earlier.

When mean tensile bond strength of group II is compared among different segments and between the subgroups using Post Hoc Tukeys test it was seen that among coronal segment it was seen that control group compared with laser values were high but not that significant. And among middle segment when control group compared with laser values were high but not significant. The reason for this were similar as explained earlier.

When mean tensile bond strength values of group I and group II were compared among subgroups at different segment using one way ANOVA test individually it was found that sandblasting group showed highest tensile bond strength values than compared with laser and control groups for all the segments. Control group showed the least tensile bond strength value for all the segments. Because in control group no surface treatments are applied. Nontreated fiber posts have a relatively smooth surface area that limits mechanical interlocking between the post surface and resin cement, and purely adhesive failure modes are commonly recorded at the post/composite interfaces. ${ }^{24}$ Among laser group and sandblasting group sand blasting showed higher tensile bond strength than laser group. Because the mechanical action of blasting probably determines the removal of the superficial layer of the resinous matrix, creating microretentive spaces on the post surface. ${ }^{19}$ It is well accepted that sandblasting with alumina particles results in increased surface roughness and surface area. After sandblasting method, the post surface area 
increased for mechanical lock of resin cement. And hence it improves the tensile bond strength values. The current studies showed that sandblasting procedures did not lead to any deformation on the posts' surface. As a result of these studies, they reported that the sandblasting method could be used safely on the post's surface. ${ }^{4}$ The use of Er:YAG laser treatment resulted in exposure of the composite matrix and damage to fibers at the surface of the FRC posts. Based on the results of the present study, these procedures cannot be recommended for clinical use due to possible weakening effects on the stability and integrity of the posts. Although laser treatment was indicated to be a promising technology in dentistry, there is still need for more research to determine appropriate parameters of laser treatment for application of this technology to dental materials. ${ }^{19}$ To obtain optimum bond strength and roughness values, adequate laser parameters should be chosen. Micro structures of the dental material also specify the ablation rate of lasers. Different laser types and different laser parameters may be used in the further studies for improving bond strength values. ${ }^{4}$

When multiple comparison of mean tensile bond strength between group I and group II and among subgroups A, B and $\mathrm{C}$ at different segments using independent $\mathrm{t}$ test, the tensile bond strength values of Calibra cement were higher compared to Rely X cement but it was not significant.

The differences in the results may be due to factors such as the handling characteristics of the adhesive system, root anatomy, tooth position, presence of coronal residual tissues; light curing technique, experience and skill of the operators. In addition, the difference in film thickness of the luting cements may contribute to the present results although all possible measures had been taken for standardization in post space preparation.

The reason for lower bond strength values at apical segment might be due to inability to produce a good surface of the intra-canal dentine wall in addition to limited access for the adhesive materials to reach the most apical part of the post space can affect the bond strength of such cements.

Calibra esthetic resin cement had an adhesive prime and bond NT which may have led to an increase in the tensile bond strength between the post and the dentin and subsequent higher mean bond strength values compared to Rely X U 200.

Some studies have affirmed that RelyX has showed a significantly lower number of penetrated dentinal tubules, lower hybrid layer thickness and the penetration of this cement into the dentinal tubules was found in only a few specimens in comparison with conventional dual-cure cements.

It should be pointed out that this study possesses some limitations. The test specimens have not had their crowns completely restored and neither thermal cycling nor mechanical stressing was applied. These factors may limit the direct application of the study results to clinical conditions. ${ }^{1}$

Most studies that tested the retention of endodontic posts were performed shortly after cementation, without any simulation of oral conditions or artificial aging. However, clinically, the dislodgement of post-retained restorations commonly occurs after several years of function, and long-term retention may be influenced by various factors such as temperature changes and dynamic mechanical loading. Therefore, in vitro tests evaluating retention of posts should aim to simulate clinical conditions by using artificial aging to more predictably mimic clinical behavior. ${ }^{2}$ So further studies on these to be done with those conditionings to know more about the materials used and to incoperate their advantages in clinical purpose.

\section{Recommendations for further study:}

1. To conduct a similar study to include more varieties of luting cement systems so as to provide a more representative result.

2. To conduct a similar study to analyse and determine features at the microscopic level contributing to bond strength using the Scanning Electron Microscope (SEM).

3. To conduct a similar study to compare the different types of adhesives, their delivery systems and the outcome to bond strength.

4. To conduct a randomized clinical trial to investigate the survival rate of different types of fibre-reinforced post cemented with various other types of luting cements.

5. To conduct a similar study using different type of laser or the same laser at different power settings for better effects to check any improvement in the bond strength. 
6. To conduct a similar study where laser is applied in the tooth surfaces along with the surface treatments on endodontic posts.

\section{Conclusion:-}

Within the limitation of the study, the following conclusions were drawn:

1. The tensile bond strength values were highest in coronal segment compared to middle and apical segments of the roots in both the groups.

2. The glass fiber post sandblasted with $110 \mu \mathrm{m} \mathrm{Al} \mathrm{O}_{3}$ with Rely-X cement had an increased significant difference compared with posts without sandblasting.

3. The glass fiber post sandblasted with $110 \mu \mathrm{m} \mathrm{Al}_{2} \mathrm{O}_{3}$ with Calibra cement had an increased significant difference compared with posts without sandblasting.

4. The glass fiber post with laser surface treatment luted with Rely- X cement had an increased tensile bond strength when compared without laser surface treatment.

5. The glass fiber post with laser surface treatment luted with Calibra cement had an increased tensile bond strength when compared without laser surface treatment.

6. The glass fiber post luted with Rely-X cement when compared between surface treatments (Laser and Sandblasting) sandblasting showed highly significant value of tensile bond strength.

7. The glass fiber post luted with Calibra cement when compared between surface treatments (Laser and Sandblasting) sandblasting showed highly significant value of tensile bond strength.

\section{Summary}

Endodontically treated teeth may require extensive coronal reconstruction and depending on the severity of the coronal tissue lose, intracanal post placement may be necessary to achieve retention to the core and restoration. Posts made of metal alloys were reported to have less retention, causes serious type of root fractures, compromise esthetics and are susceptible to corrosion. As alternative, fiber-reinforced composite (FRC) posts were developed with intensive research interest. ${ }^{1}$ Many in vitro studies have investigated various factors that affect retention of a post, include length, design, diameter, and the surface treatments. Chemical interaction that forms the bond between fiber post and core is not sufficient enough to withstand the occlusal stresses. Surface pre-treatment of fiber post is the common method employed to improve the adhesion property of material. Innumerable number of post cement materials are available in the market claiming various levels of efficiency. The credibility of these cements when clubbed with surface treated posts though has not been analyzed in detail. Hence this in vitro study evaluation and comparison of the tensile bond strength of two different luting agents on an endodontic fiber post after two different surface treatments was conducted. The aim of the study was to evaluate and compare tensile bond strength of two different luting agents on an endodontic fiber post after two different surface treatments.

Sixty mandibular first premolars were selected and cut perpendicular to the long axis after marking $14 \mathrm{~mm}$ from the apex of the tooth towards the incisal surface. Root canals were prepared using step-back technique and obturated with gutta-percha using lateral condensation. Post space preparation was done using Peeso Reamers. The roots were divided into 2 experimental groups based on the luting cements and further divided into 3 subgroups according to surface treatments used $(n=10)$. (10 control group, 10 sandblasted group, 10 laser treated group). Posts were surface treated according to the groups.Resin luting agents (Rely X U200 \& Calibra) were mixed according to manufacturer's instruction and then placed in the post spaces and the posts were cemented to the prepared specimens. Each root was cut horizontally, and three 2 -mm-thick root segments (1 apical, 1 middle, and 1 cervical) were prepared. The specimens were later stored in saline for $24 \mathrm{hrs}$ at $37^{\circ} \mathrm{C}$. Using a push-out test, the bond strength between post and dentin was measured using a universal testing machine. The result obtained was tensile bond strength values was superior in coronal compared to middle and apical segments of the roots in both the groups. The mean tensile bond strength values of group $\mathrm{C}$ were highly significant for both group I and group II. The mean tensile bond strength values of group II was better than group I after both the surface treatments but it was not highly significant.

\section{And within the limitation of the study, the following conclusions were drawn:}

1. The tensile bond strength values was highest in coronal segment compared to middle and apical segments of the roots in both the groups.

2. The glass fiber post sandblasted with $110 \mu \mathrm{m} \mathrm{Al} \mathrm{O}_{3}$ with Rely-X cement had an increased significant difference compared with posts without sandblasting. 
3. The glass fiber post sandblasted with $110 \mu \mathrm{m} \mathrm{Al}_{2} \mathrm{O}_{3}$ with Calibra cement had an increased significant difference compared with posts without sandblasting.

4. The glass fiber post with laser surface treatment luted with Rely- X cement had an increased tensile bond strength when compared without laser surface treatment.

5. The glass fiber post with laser surface treatment luted with Calibra cement had an increased tensile bond strength when compared without laser surface treatment.

6. The glass fiber post luted with Rely-X cement when compared between surface treatments (Laser and Sandblasting) sandblasting showed highly significant value of tensile bond strength.

7. The glass fiber post luted with Calibra cement when compared between surface treatments (Laser and Sandblasting) sandblasting showed highly significant value of tensile bond strength.

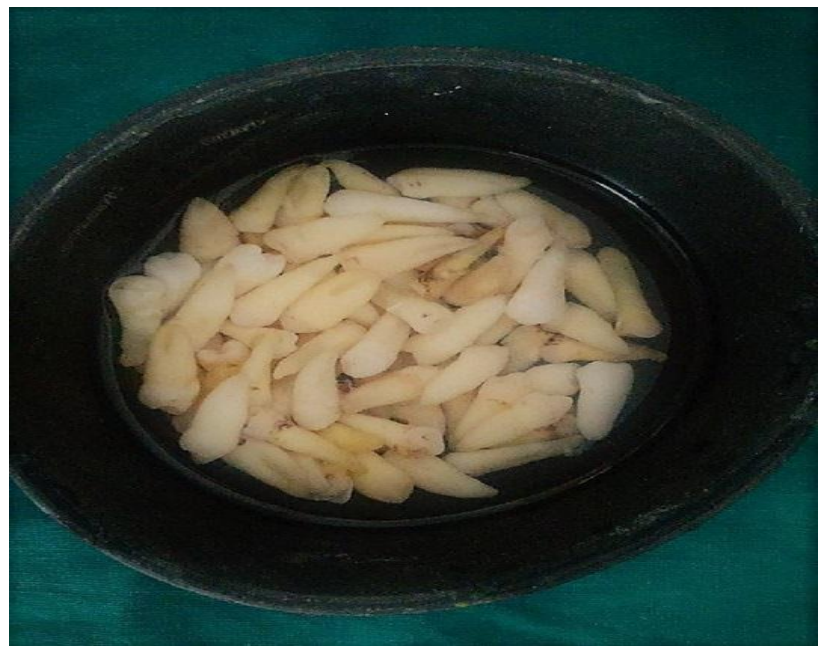

Figure 3:-Specimen preserved in saline

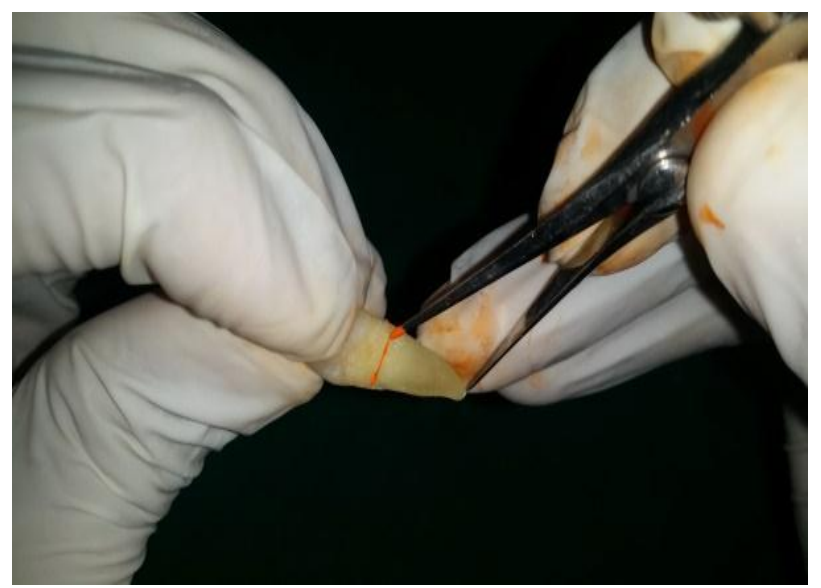

Figure 4:-Marking $14 \mathrm{~mm}$ from the apex of root 


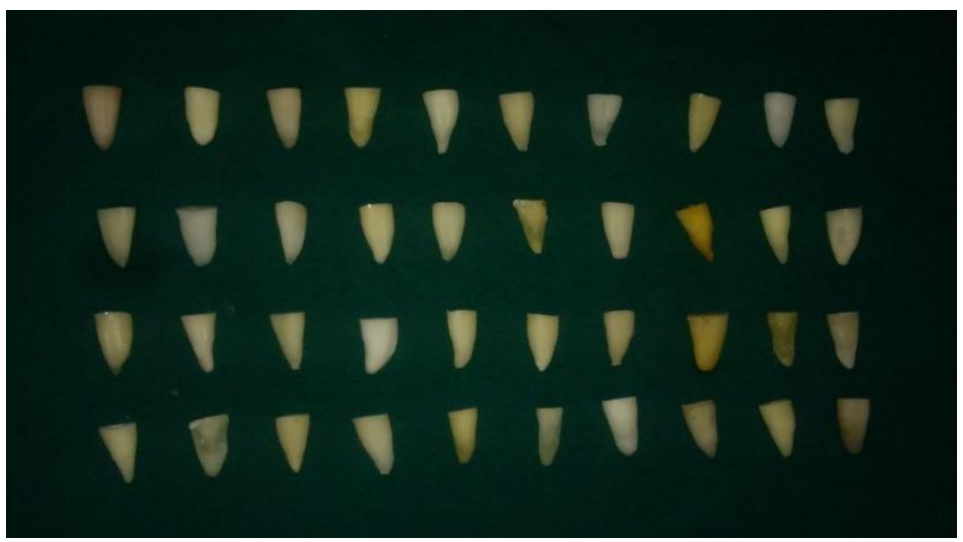

Figure 5:-Sectioned specimens

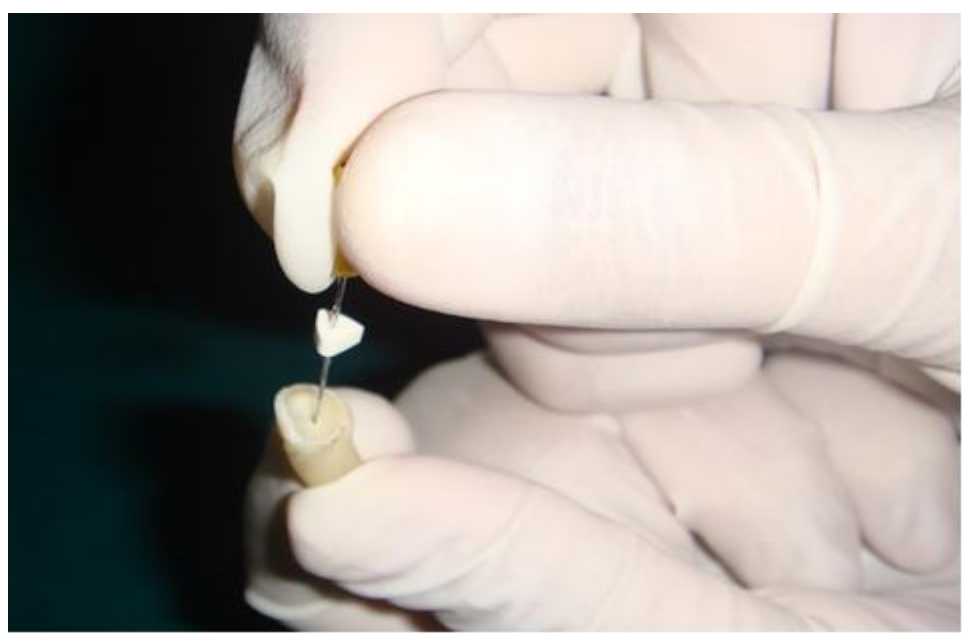

Figure 6:-Root canal treatment using $\mathrm{K}$-file after marking $13 \mathrm{~mm}$

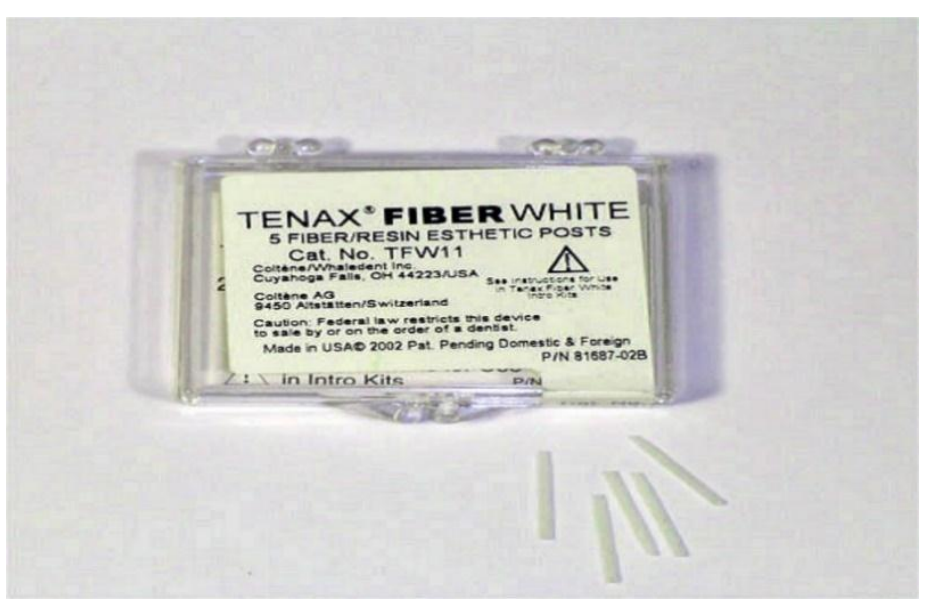

Figure 7:-Tenax fiber post 


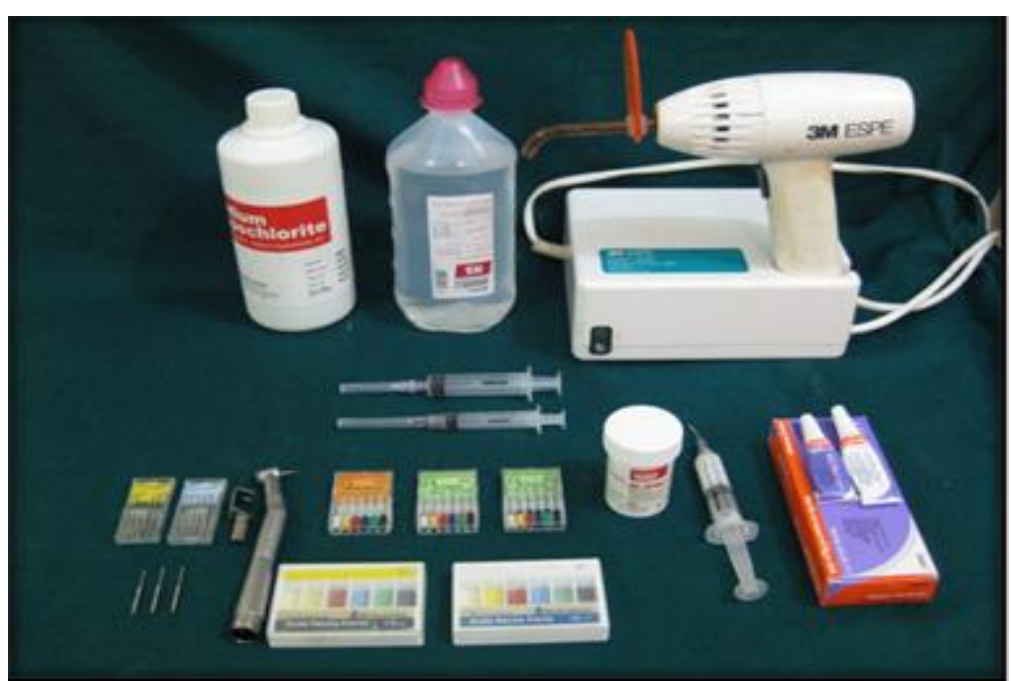

Figure 8:-5.25\% NaOCl, 15\% EDTA, gutta-percha points, Airoter, K-files and saline AH sealer, LED curing unit.

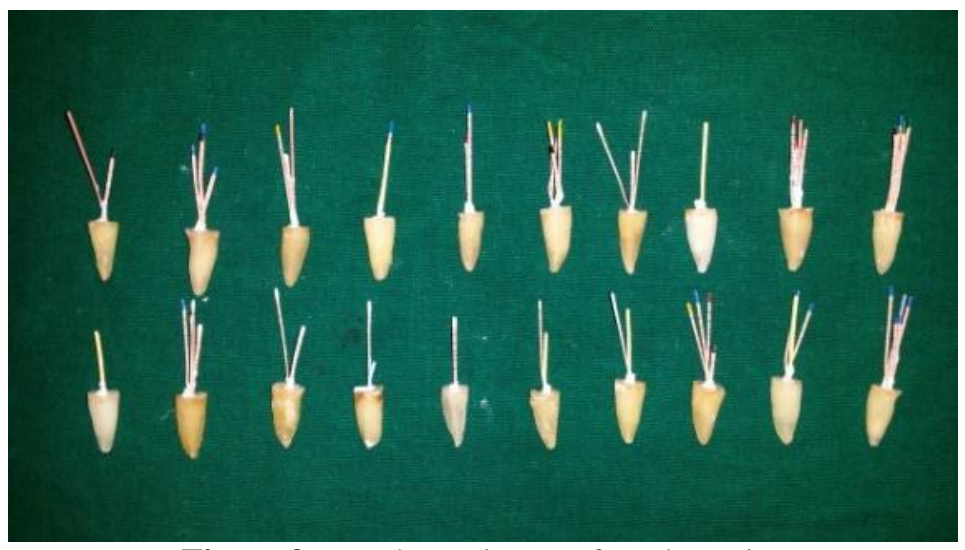

Figure 9:-Tooth specimens after obturation

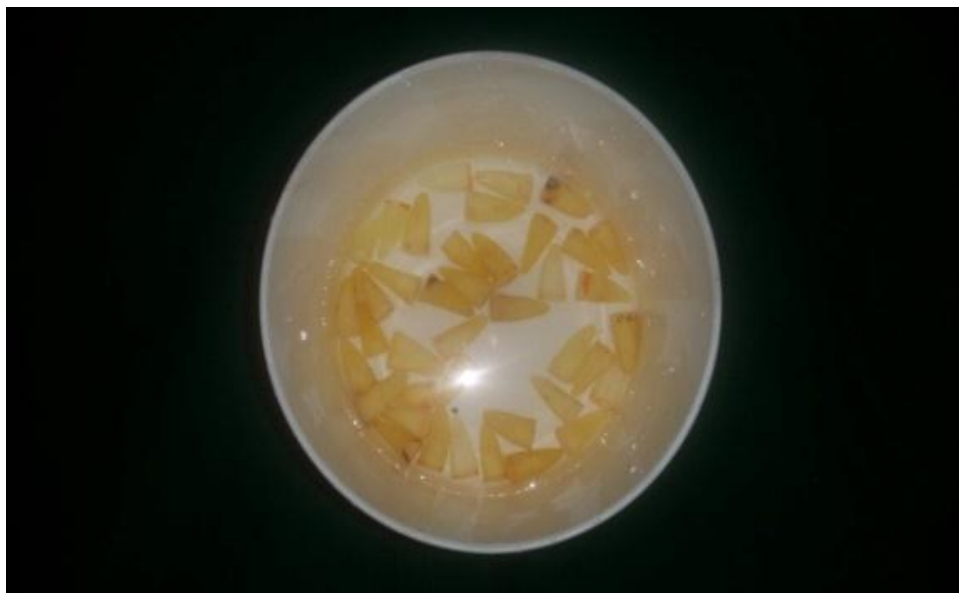

Figure 10:-Specimens stored in distilled water after root canal treatment 


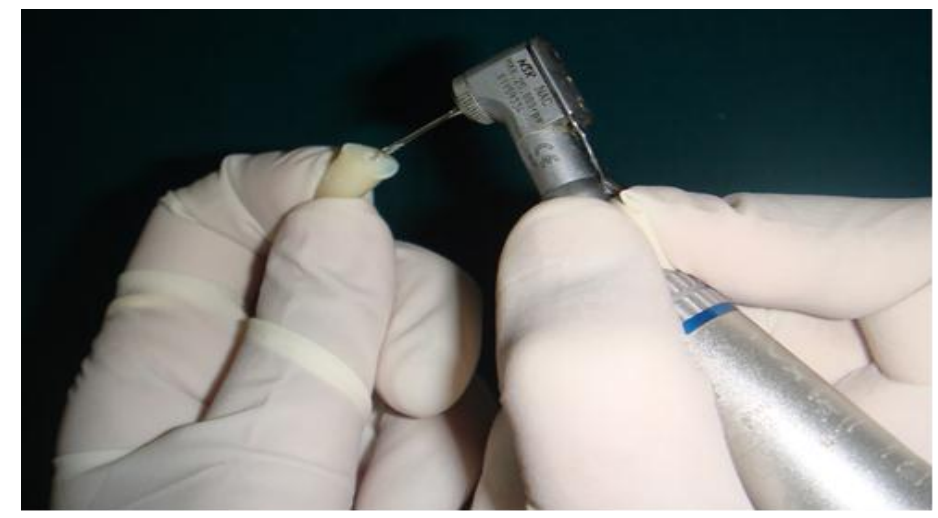

Figure 11:-Post space preparation on specimen

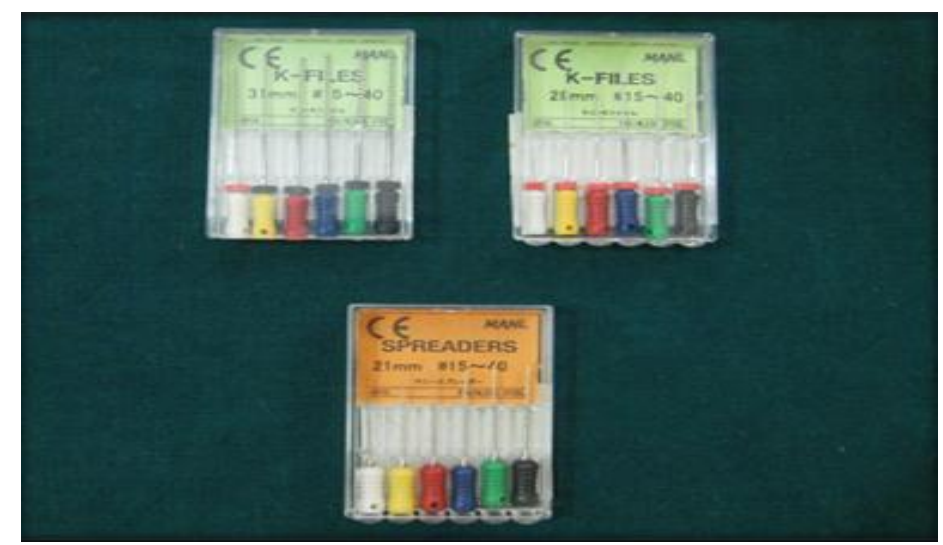

Figure 12:-Hand k files and spreaders

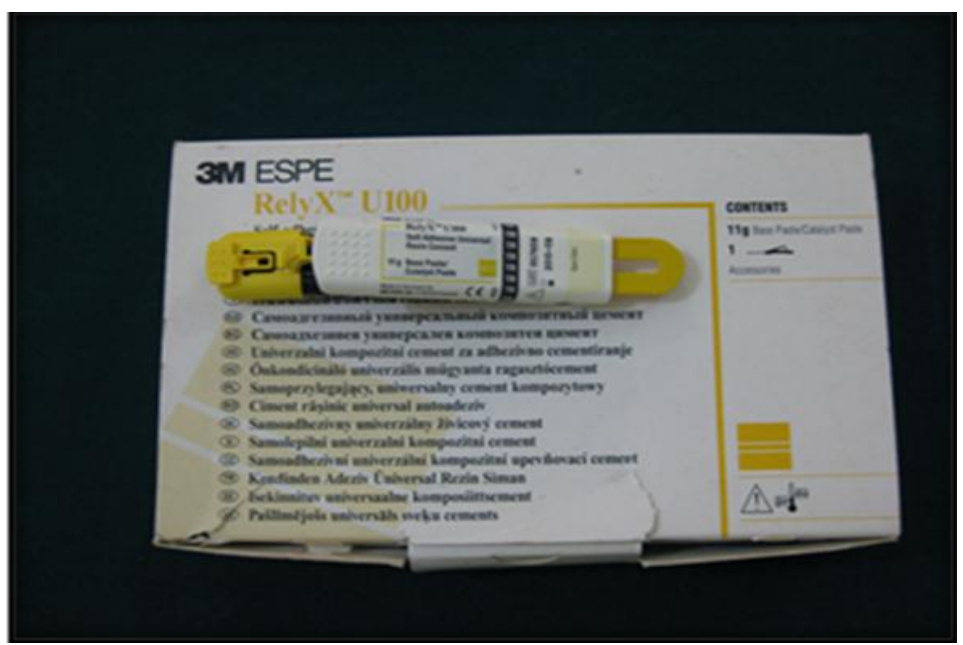

Figure 13:-Rely X cement 


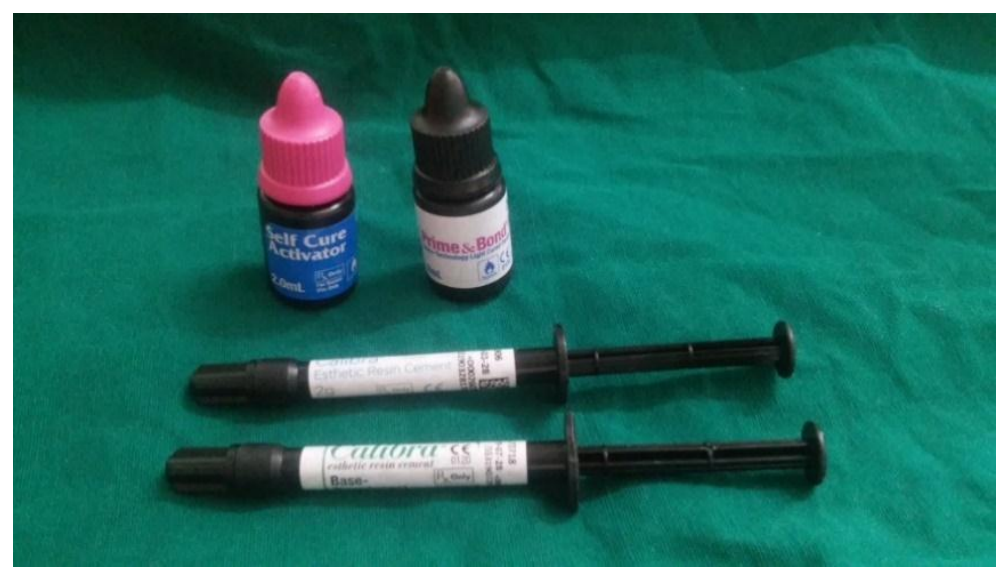

Figure 14:- Calibra cement

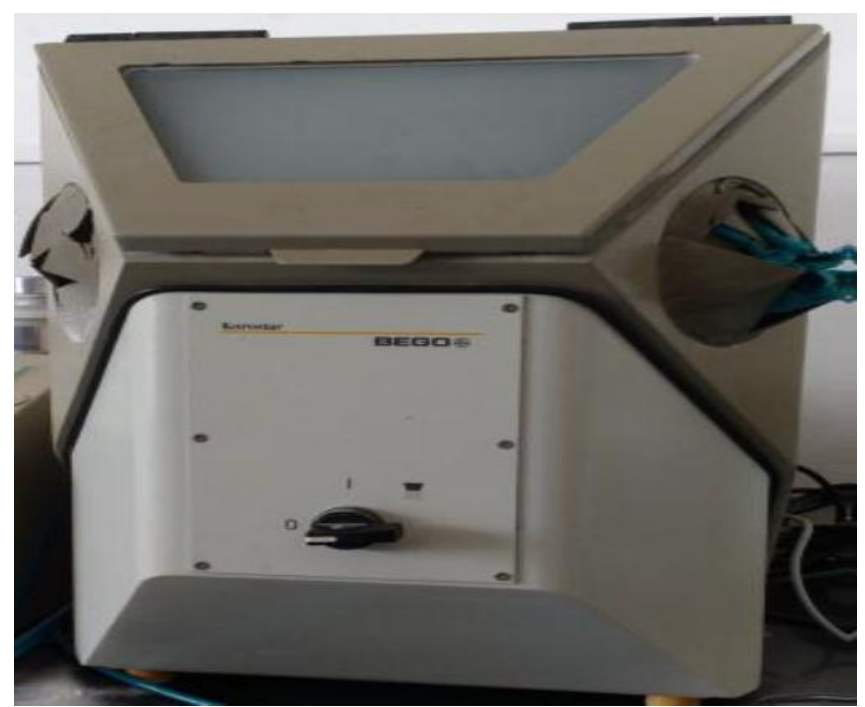

Figure 15:- Sandblasting unit

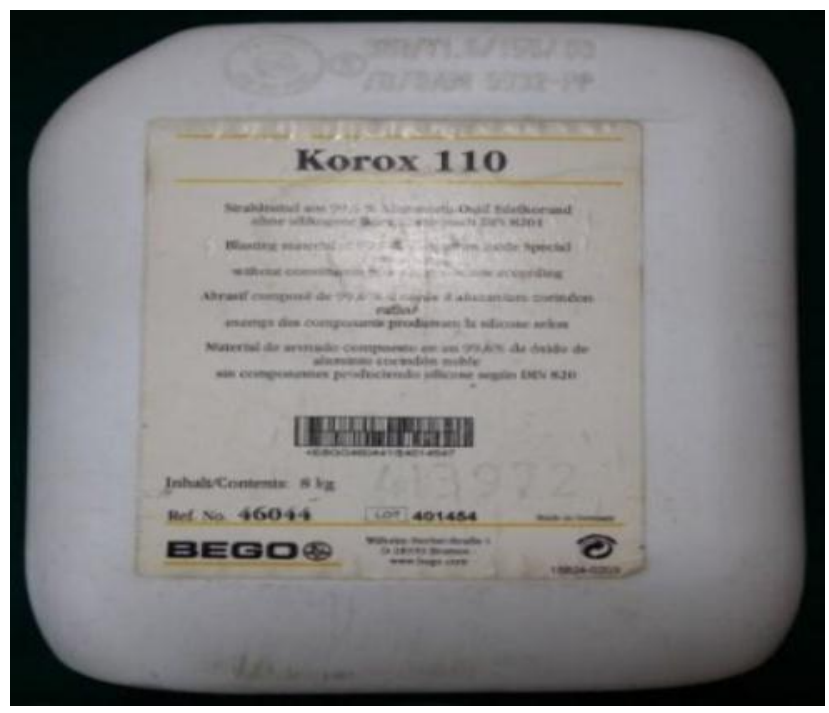

Figure 16:-110 $\mu \mathrm{m} \mathrm{Al}_{2} \mathrm{O}_{3}$ 


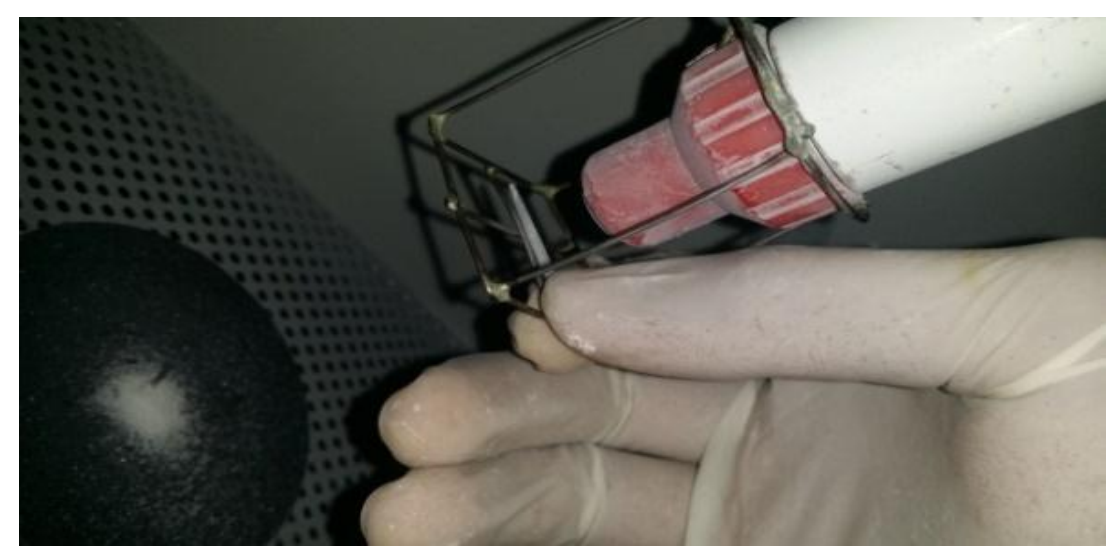

Figure 17:-Sandblasting of posts with $110 \mu \mathrm{m} \mathrm{Al}_{2} \mathrm{O}_{3}$

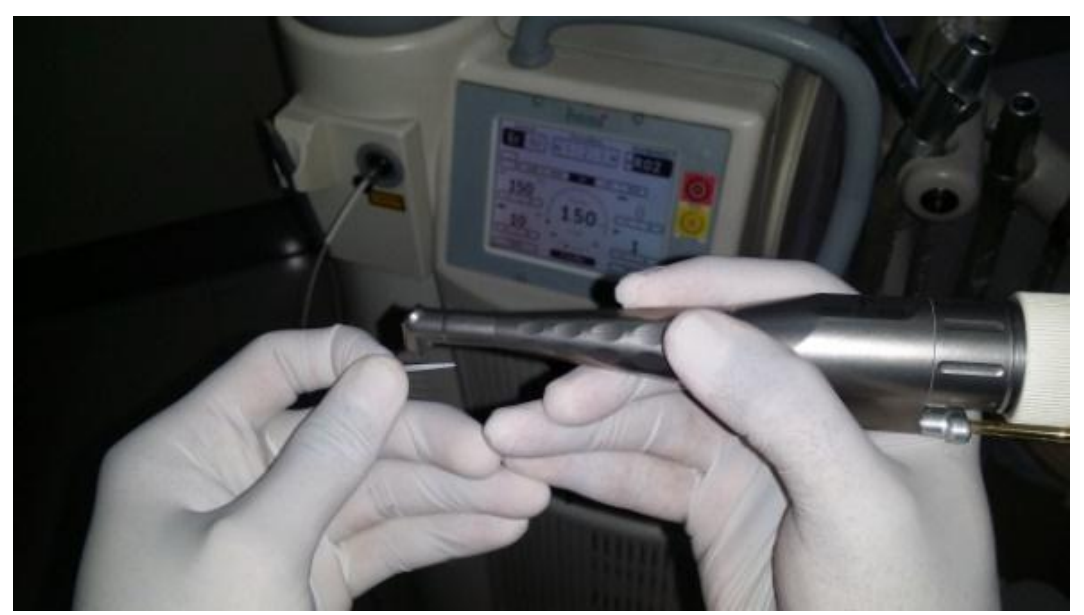

Figure 18 -Laser treatment of posts with Er:YAG laser

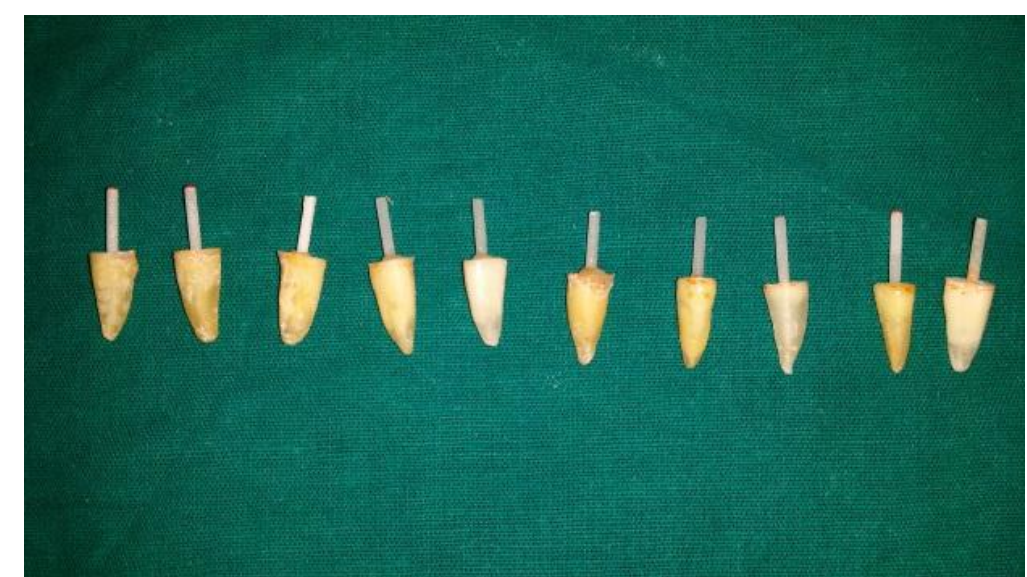

Figure 19:-Specimens after post cementation (Rely X and Calibra cements) 


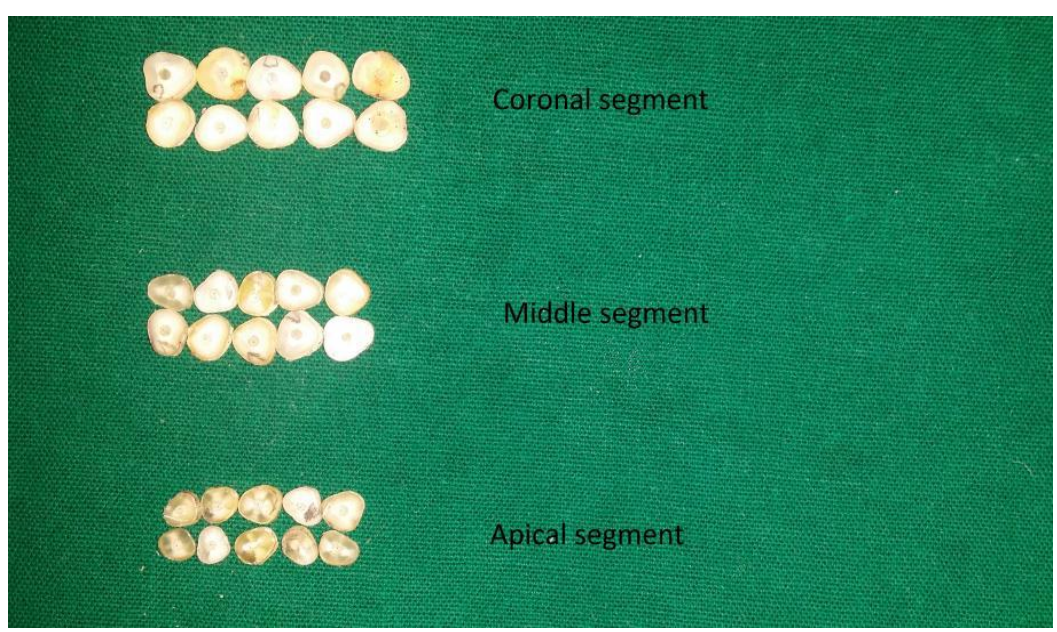

Figure 20:-Sectioned Specimens

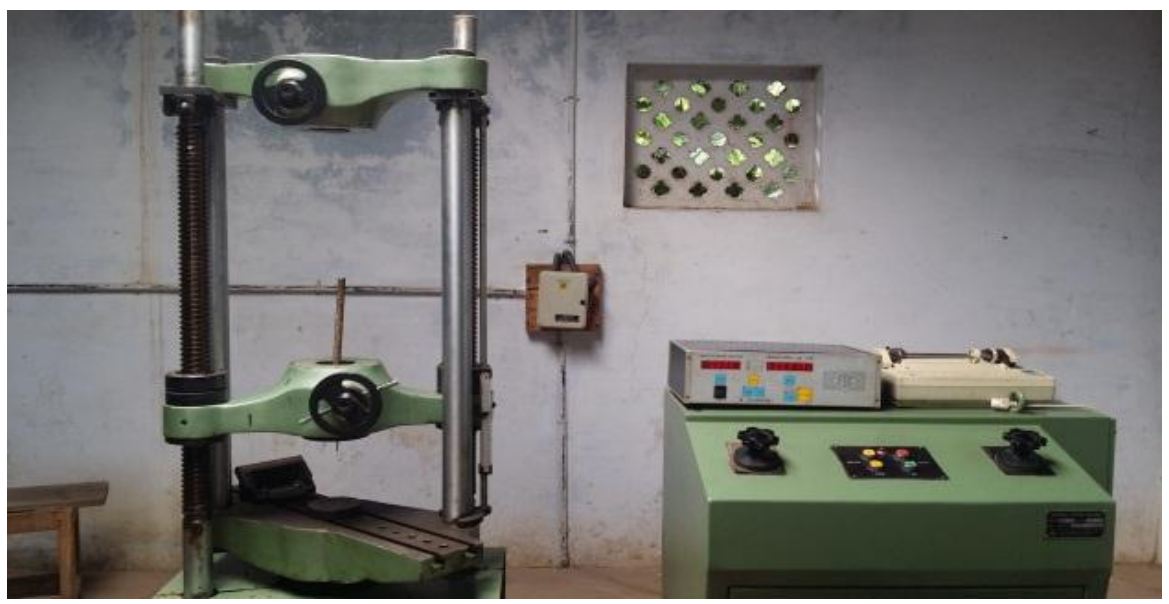

Figure 21:-Universal testing machine

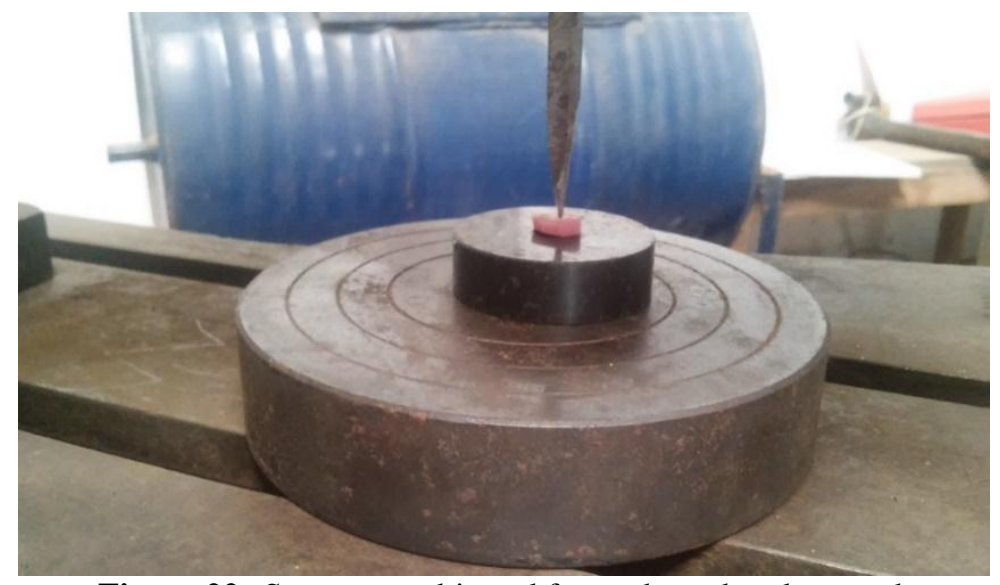

Figure 22:-Segments subjected for push out bond strength 


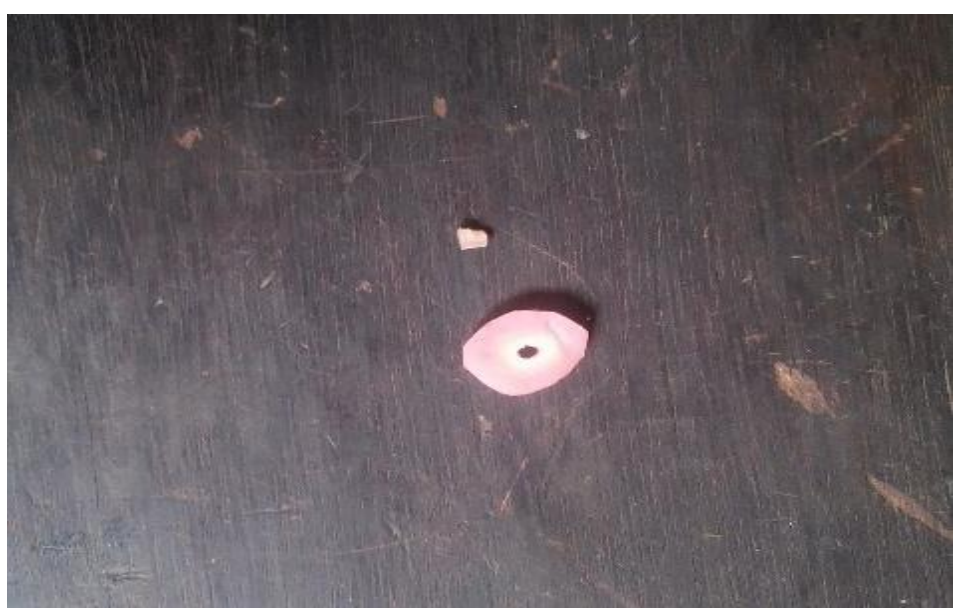

Figure 23:-Specimens after push out bond strength

\section{Bibliography:-}

1. Gomes G M, Gomes O M M, Reis A, Carlos, Gomes J C, Loguercio A D, Calixto A L. Regional bond strength to root canal dentin of fiber posts luted with three cementation system. Braz Dent J 2011; 22(6):460-467.

2. Balbosh A, Kern M. Effect of surface treatment on retention of glass-fiber endodontic posts. J Prothet Dent 2006; 95:218-23.

3. Shori D, Pandey S, Kubde R, Rathod Y, Atara R, Rathi S. To evaluate and compare the effect of different post surface treatments on the tensile bond strength between fiber posts and composite resin. J Int Oral Health. 2013 Oct; 5(5): 27-32.

4. Arslan H, Kurklu D, Ayrancı L B, Barutcigil C, Y1lmaz C B, Karatas E, Topcouglu H S. Effects of post surface treatments including Er:YAG laser with different parameters on the pull-out bond strength of the fiber posts. $\mathrm{J}$ Lasers Med Sci 2014 Sep; 29(5):1569-1574.

5. Calixto L R, Bandeca M C, Clavijo V, Andrade M F, Vaz L G, Campos E A. Effect of resin cement system and root region on the push out bond strength of a translucent system. J Oper Dent 2012;37 (1),80-86.

6. Gaston B A, West L A, Liewehr F R, Fernades C, Pashley D H. Evaluation of regional bond strength of resin cement to endodontic surfaces. J Endod 2001; May 27(5):321-324.

7. Pest B L, Cavalli G, Bertani P, Gagliani M. Adhesive post endodontic restorations with fiber posts: push out tests and SEM observation. J Dent Mater. 2002; 18:596-602.

8. Furuya Y, Huang SH, Takeda Y, Fok A, Hayashi M. Fracture strength and stress distributions of pulpless premolars restored with fiber posts. J Dent mater. 2014 Nov 28;33(6):852.

9. Graiff L, Rasera L, Calabrese M, Vigolo P. Bonding effectiveness of two adhesive luting cements to glass fiber posts: pull-out evaluation of three different post surface conditioning methods.. Int J Dent.2014 1-8

10. Aggarwal R, Gupta S, Tandan A, Gupta NK, Dwivedi R, Aggarwal R. Comparative evaluation of fracture resistance of various post systems using different luting agents under tangential loading J Oral Biol Craniofac Res. 2013 May 1;3(2):63-7.

11. Standlee JP, Caputo AA, Hanson EC. Retention of endodontic dowels: effects of cement, dowel length, diameter, and design. J Prosthet Dent 1978 Apr;39(4):400-5.

12. Purton DG, Love RM, Chandler NP. Rigidity and retention of ceramic root canal posts. J Operat dentistry. 2000 May 1;25(3):223-7.

13. Qualtrough AJ, Chandler NP, Purton DG. A comparison of the retention of tooth-colored posts. J Res Dent 2003 Mar 1;34(3).

14. Mallmann A, Jacques LB, Valandro LF, Muench A. Microtensile bond strength of photoactivated and autopolymerized adhesive systems to root dentin using translucent and opaque fiber-reinforced composite posts. J prosthet Dent. 2007 Mar 1;97(3):165-72.

15. Bonchev A, Radeva E, Tsvetanova N. Fiber Reinforced Composite Posts-A Review of Literature. Int J Sci Res 2017;6(10):1887-93.

16. Choi Y, Pae A, Park EJ, Wright RF. The effect of surface treatment of fiber-reinforced posts on adhesion of a resin-based luting agent. J Prosthet Dent 2010 Jun 1;103(6):362-8.

17. Valdivia AD, Novais VR, Menezes MD, Roscoe MG, Estrela C, Soares CJ. Effect of surface treatment of fiberglass posts on bond strength to root dentin. Braz Dent J 2014;25(4):314-20. 
18. Aktemur Türker S, Uzunoğlu E, Yılmaz Z. Effects of dentin moisture on the push-out bond strength of a fiber post luted with different self-adhesive resin cements. Restor Dent Endod 2013 Nov 1;38(4):234-40.

19. Kurt M, Güler AU, Duran İ, Uludamar A, İnan Ö. Effects of different surface treatments on the bond strength of glass fiber-reinforced composite root canal posts to composite core material. J Oral Sci 2012 Mar 1;7(1):20-5.

20. Mosharraf R, Ranjbarian P. Effects of post surface conditioning before silanization on bond strength between fiber post and resin cement. J Adv Prosthodont 2013 May 1;5(2):126-32.

21. Poplai G, Jadhav S, Hegde V. Surface analysis of glass fiber posts after pretreatment ER: YAG laser: An SEM study. J Dent Lase. 2013 Jul 1;7(2):72.

22. Er Ö, Kılıç K, Kılınç Hİ, Aslan T, Sağsen B. Evaluation of the resin cement thicknesses and push-out bond strengths of circular and oval fiber posts in oval-shapes canals. J Adv Prosthodont 2015 Feb 1;7(1):15-20.

23. Kalkan M, Usumez A, Ozturk AN, Belli S, Eskitascioglu G. Bond strength between root dentin and three glassfiber post systems. J Prosthet Dent. 2006 Jul 1;96(1):41-6.

24. Goracci. C, Grandini. S, Bossu. M, Bertelli. E and Ferrari. M. Laboratory assessment of the retentive potential of adhesive posts. J Dent. 2007 Nov;35(11):827-35. 2007 Sep 4.

25. Bitter. K, Priehn. K, Martus. P and Kielbassa. A. M. In- vitro evaluation of push out bond strengths of various luting agents to tooth colored posts. J Prosthet Dent. 2006 Apr;95(4):302-10.

26. Yahya NA. Effect of luting cement to push-out bond strength of fibre reinforced post. Int Endod J 2018 Jun 30;15(1):11-9.

27. Das AK, Muddugangadhar BC, Amarnath GS, Garg A, Kumar U, Rao TP. Comparative evaluation of push out bond strength of a fiber post system using four different resin cements: An in-vitro study. Journal of international oral health: J Int Oral Health . 2015;7(Suppl 1):62.

28. Kırmalı Ö, Üstün Ö, Kapdan A, Kuştarcı A. Evaluation of Various Pretreatments to Fiber Post on the Push-out Bond Strength of Root Canal Dentin. J Endod 2017 Jul 1;43(7):1180-5. 